Integrative

Medicine
Integr Med Int 2015;2:90-128

DOI 10.1159/000441070
Published online: October 14, 2015

This article is licensed under the Creative Commons Attribution-NonCommercialNoDerivatives 4.0 International License (CC BY-NC-ND) (http://www.karger.com/Services/ OpenAccessLicense). Usage and distribution for commercial purposes as well as any distribution of modified material requires written permission.

\title{
BIT's 2nd Annual World Congress of High-Tech Acupuncture and Integrative Medicine
}

Hangzhou, May 2015

\author{
Guest Editor \\ G. Litscher, Graz
}

Part I:

Selected Abstracts - Keynote and Main Lectures

Part II:

Selected Abstracts - Poster Presentations 


\section{Selected Abstracts - Keynote and Main Lectures}

\author{
High-Tech Acupuncture and Integrative \\ Medicine - Current Research and Future \\ Aspects \\ Gerhard Litscher \\ Professor, President of ISLA (Science and Research), \\ German Vice-President of the DCFG-TCM, Medical \\ University of Graz, Austria \\ E-Mail gerhard.litscher@medunigraz.at
}

\section{Abstract}

Clinical and basic research on high-tech acupuncture and integrative medicine has been performed all over the world during the last decades, using a broad spectrum of innovative biomedical engineering methods. Acupuncture has been used for medical treatment for thousands of years. Using electroacupuncture, needle or laser needle stimulation and modern biomedical techniques, it was possible for the first time to quantify changes in biological activities caused by acupuncture. This keynote lecture focuses on the latest innovative aspects in the development of high-tech acupuncture. Special emphasis is given to new acupuncture and integrative medicines innovations, e.g. teleacupuncture and laser therapy in experimental and clinical studies.

The studies are supported by the Austrian Federal Ministries of Science, Research, and Economy and of Health and by Eurasia Pacific Uninet.

\section{Biography}

Prof. Litscher is the head of the Research Unit for Complementary and Integrative Laser Medicine, of the Research Unit of Biomedical Engineering in Anesthesia and Intensive Care Medicine, and of the TCM Research Center Graz at the Medical University of Graz, Doctor of technical and Doctor of medical sciences, international lectures, about 600 scientific publications, partly on basic acupuncture research, author and/or editor of 12 books, currently editor-in-chief and/or member of the editorial board of more than 35 international scientific journals (e.g. Editor-in-chief of the Internet Journal of Alternative Medicine (IJAM), medicines, Integrative Medicine International, Associate Editor for Medical Acupuncture, Associate Editor of the Journal of Acupuncture and Meridian Studies (JAMS), one of the editors and lead guest editor of Evidencebased Complementary and Alternative Medicine (eCAM), and Associate Editor of BMC Complementary and Alternative
Medicine). Prof. Litscher's special interests are computerbased High-Tech Acupuncture Research and Neuromonitoring. He is University Professor at the Medical University of Graz and Visiting/Guest Professor at eight institutions and universities in Asia. He is President for Science and Research of ISLA-transcontinental (International Society for Medical Laser Applications) and German Vice-President of the German-Chinese Research Foundation for Traditional Chinese Medicine (DCFG-TCM).

\section{Brain Imaging-From Molecule to Nerve Tractography by Ultra High Field MRI \\ Zang-Hee Cho \\ Professor, Seoul National University, South Korea \\ E-Mail zhcho36@gmail.com}

\section{Abstract}

Brain Imaging with new PET-MRI, using High resolution PET and Ultra High Field 7.0T Magnetic Resonance Imaging (MRI) and their applications to Brain Research, especially to the areas of neuropsychiatry, neurosurgery and neuroscience, and acupuncture will be discussed. Among the interesting topical areas, applications of the high resolution brain PET (HRRT) and the ultra high field MRI (7.0T) will be highlighted, especially focused on the acupuncture research.

With high field MRI, such as the 7.0T MRI, one can new visualize the high resolution tractography hitherto unable to do with existing MRI systems. Together with molecular imaging using Positron Emission Tomography (PET), that is the brain dedicated PET-MRI fusion system developed recently, now, it is possible to visualize molecular mechanisms quantitatively.

\section{Biography}

Prof. Zang-Hee Cho received $\mathrm{PhD}$ from Uppsala University (Sweden) in 1966 and has been faculty at the University of Stockholm and University of California-Los Angeles. In 1979, Dr. Cho moved to Columbia University as a Professor of Radiology (Physics). Since 1985, Dr. Cho was the Professor of Radiological Science at University of California at Irvine. From 2005, Prof. Cho served as University Professor and Director of the Neuroscience Research Institute, Gachon 
University of Medicine \& Science, Incheon, till he joined as a Distinguished Research Fellow at the Adavanced Institute of Convergence Technology (AICT), Seoul National University, Seoul, Korea.

Professor Cho has been a pioneer in Positron Emission Tomography (PET) and Magnetic Resonance Imaging since the inception of the computerized tomography (CT) in 1972. He was the first one who pioneered world's first 'Ring PET', the first molecular imaging device, in 1975.

More recently, Professor Cho pioneered the first PET-MRI (Proteomics 2008) demonstrating that in-vivo human submillimeter high resolution molecular imaging is possible and published over one hundred neuroscience and related scientific publications. Prof. Cho has more than 300 peer reviewed scientific publications covering from nuclear physics to neuroscience and published 3 graduate level text books.

Among the numerous honors and awards, Professor Chowas elected as a member of the US National Academy of Sciences-Institute of Medicine in 1997.

\section{Telling What's Right and Efficient - High-Tech TCM Needs Double-Blinding, Objective Assessments and Adequate Controls}

\author{
Henry Johannes Greten \\ Professor, Heidelberg School of Medicine, Germany \\ E-Mail heidelbergschool@aol.com
}

\section{Abstract}

The future of Chinese Medicine and its recognition will largely depend on the quality of related research. Many western countries have introduced acupuncture and partly TCM. However, skepticism and even rejection is often still observed within the western academic community. One way to overcome this attitude consists of upgrading research methodology. 1) Objective assessments of effects by physically measurable parameters to evaluate acupuncture effects ('high-tech acupuncture') is a necessity, as so-called 'fluffy' data from clinical scores are often insufficiently convincing. 2) One of the crucial questions is whether animal studies and studies made under laboratory conditions may be transferred to clinical medicine. We have found a way to doubleblind acupuncture so as to control possible suggestive effects in RCTs. 3) The problem of adequate controls in acupuncture studies is still not really solved in most of the studies published. Control interventions that are exactly as invasive as the verum interventions should be scientific standard. We also developed adequate controls in other fields of TCM such as placebo qigong. The results of these studies imply the necessity of a major upgrade of the role and quality of Chinese medical theory as a prerequisite for the integration in western medical healthcare systems and research.

\section{Biography}

Henry Johannes Greten, MD, PhD, is Professor for Chinese Medicine as Applied Neurophysiology of the University of Porto and Director of the renowned Heidelberg School of Chinese Medicine. He ranks No. 1 among the 100 best physicians in Germany in the category of naturopathy (Tina No. 39/2009). His major contributions to research into Chinese Medicine include the development of the physiological model of a TCM as system theory (Heidelberg Model), the introduction of double-blinding into acupuncture research and placebo qigong. Numerous medical publications, nine textbooks including the standard textbook 'Kursbuch Traditionelle Chinesische Medizin' (Thieme) and 'Checkliste Chinesische Phytotherapie' (Hippokrates). President, German Society of TCM, President, ISCMA, Director, Heidelberg School of Chinese Medicine, Vice-President, Debakey Living Heart Alliance, Houston, President, Scientific Board, SAPMEC, President, Sino-German Research Foundation.

\section{Neuro-Rehabilitation and Traditional Chinese Medicine at the Private Clinic in Lassnitzhoehe \\ Walter Kreuzig \\ Medical Director, Private Clinic Lassnitzhoehe, Lassnitzhoehe, Austria \\ E-Mail w.kreuzig@sanlas.at}

\section{Abstract}

In private clinic Lassnitzhöhe, Austria, neurologic rehabilitation has been performed for more than twenty years. The goals of neurologic rehabilitation are to regain lost abilities, to achieve social as well as work related re-integration and to avoid or reduce the need of care. To achieve these goals we need team work, done by many specialists and based on new evidence of brain function, plasticity and motor learning. The presentation will focus on the rehabilitation of stroke, which is a worldwide burden and the most common reason of disability of elderly people. The different concepts, the computerized training devices used in the clinic and the supportive cooperation with TCM, performed by a Chinese specialist, will be demonstrated.

\section{Biography}

Prim. Dr. Walter Kreuzig was born on May 31, 1955. He studied in Karl Franzens University School of Medicine from 1973-1979 in Graz. Then, he acted as Internships, rotations in state hospitals for three years. In 1987, Dr. Kreuzig obtained the accreditation as Neurologist and Psychiatrist. From 1982-1987, he stayed in Krankenhaus der Barmherzigen Brüder, for specialty training in Neurology and Psychiatry. After 1987, Dr. Kreuzig changed to Landesnervenklinik Sigmund Freud, his interests is stroke treatment. Implementation of a stroke unit and a special unit for early neurologic 
rehabilitation. 2004 to date, Dr. Kreuzig is Medical director of Privatklinik Lassnitzhöhe. Specialized in neurologic rehabilitation, esp. stroke.

\section{Acupuncture Effects on PNEI, Psycho-Neuro-Endocrine-Immune System New Trends}

\author{
Konstantina Theodoratou \\ Lecturer at ATC, Acupuncture Training Center, \\ Fellowship Biomedical Research Foundation of the \\ Academy of Athens, ICMART President, International \\ Council of Medical Acupuncture and Related \\ Techniques, SAMAG President, Scientific Association of \\ Medical Acupuncture in Greece, Greece \\ E-Mail docdia@hotmail.com
}

\section{Abstract}

It is more important to know what sort of person has a disease than to know what sort of disease a person has Hippocrates. The development of PNEI, Psycho-Neuro-Endocrine-Immune System results in a change of perspective, from a specific target to a unified point of view, relating to the interpretation of the biological functions of the body. A key point is the recognition of the importance of continuous interaction between cells, organs and systems based on a large number of messenger molecules. It is well known that acupuncture acts on each one of these levels separately. Extensive evidence shows that there are multidirectional communication pathways among the neural, endocrine, and immune systems and we will study the possible effects of acupuncture on PNEI system. For example, acupuncture influences immune cells specific to catecholamine release during sympathetic nervous system activation and other receptors specific to corticosteroids release by the limbichypothalamic-pituitary-adrenal axis. The above constitute the primary driving forces of the stress response that produce an inhibitory effect on immune function. Our aim is to extract evidence from recent data and reach a conclusion about acupuncture effectiveness on modulating biological responses associated with PNEI system.

\section{Biography}

Konstantina after completing her medical degree at the University of Athens and practising Western Medicine as a family doctor, went to China to study Acupuncture in Tianjin and Beijing Universities. She further deepened her studies in Guangzhou (Canton) University where she obtained her MSc in Acupuncture. She has also specialised in Medical Psychology, Addiction Counseling and Cognitive Neuroscience at the University of Athens. She has recently participated in research, mostly concerning behavior patterns in relation to Acupuncture conducted by the Biomedical Research Foundation of the Academy of Athens, published papers and lectured in various congresses. Currently she lectures at the
Greek Institutes of Acupuncture. Konstantina is the President of ICMART, International Council of Medical Acupuncture and Related Techniques and President of the Scientific Association of Medical Acupuncture in Greece.

\section{Dense Cranial Electroacupuncture Stimulation, a Novel Brain Stimulation Therapy for Major Depression: Rationale and Evidence from Clinical Trials}

\author{
Zhang-Jin Zhang
}

Professor, School of Chinese Medicine, The University of Hong Kong, Hong Kong, China

E-Mail zhangzj@hku.hk

\section{Abstract}

Dense cranial electroacupuncture stimulation (DCEAS) is a novel brain stimulation mode in which electrical stimulation is delivered on dense forehead acupoints innervated by the trigeminal sensory pathway. Neuroanatomical evidence suggests that, compared to the spinal-supraspinal pathways, the trigeminal sensory pathway has much intimate connections with the brainstem reticular formation, in particular the dorsal raphe nuclei and the locus coeruleus, both of which are the major resources of serotonergic $(5-\mathrm{HT})$ and noradrenergic neuronal bodies, respectively, and play a pivotal role in processing perception, emotion, and sleep. This has led to the hypothesis that direct stimulation on forehead acupoints in the trigeminal territory could more efficiently produce therapeutic response in neuropsychiatric disorders. We have completed 4 clinical trials of DCEAS as adjunctive treatment in patients with major depression, postpartum depression and post-stroke depression. These clinical trials demonstrated that DCEAS is considerably beneficial in alleviating the severity of depression symptoms, accelerating onset of antidepressant action, enhancing clinical response and reducing antidepressant doses taken. This talk will report the results of these trials and discuss the rationale of DCEAS. [The studies were supported by HMRF (06070831), GRF/RGC (786611) and HA funds].

\section{Biography}

Professor Zhang Zhang-Jin is a Tenured Full Professor and Associate Director of School of Chinese Medicine, Honorary Professor of Psychiatry, Family Medicine and Primary Care and Anatomy in the University of Hong Kong (HKU). He serves Vice-President of the Hong Kong Association for Integration of Chinese-Western Medicine, Deputy Director of Specialty Committee of Mental Diseases of World Federation of Chinese Medicine Societies (WFCMS), and Deputy Director of Sleep Medicine Specialty Committee of WFCMS. He received his Chinese medicine, acupuncture and Western medicine training and earned his $\mathrm{PhD}$ in neuroscience in China. During 1994-2006, Professor Zhang moved to the United States to continue his research work in psychopharma- 
cology and psychiatry at Vanderbilt University and then Uniformed Services University of the Health Sciences (USUHS). His research interest focuses on developing novel treatment strategies from Chinese medicine and acupuncture for major psychiatric disorders, including depression, anxiety, dementia, and schizophrenia. He is also interested in exploring neural mechanisms of acupuncture. Professor Zhang has published numerous basic and clinical research papers of acupuncture and herbal medicine.

\section{Research for Fire Needling Instruments in the Practice of Acupuncture}

\section{Lin-Peng Wang}

Vice President, China Association of Acupuncture and

Moxibustion, China

E-Mailwlp5558@sina.com

\section{Abstract}

Fire-needle puncture is one popular type of the traditional Chinese acupuncture therapy. It is applied by a specific kind of needle instrument that heating to red-hot by fire and then pierce into acupoints. Fire-needle therapy, with a long history of clinical practice, shows outstanding effect for a large range of diseases and symptoms. In recent years, more than 160 papers reported the effect of fire-needle therapy for a variety of diseases. However, limitations of this therapy have been shown because that the elemental character of the instrument remains unclear. To develop the therapy of fire needle further, scientific research and standardized guideline of fire needling should be implemented. Hence, we conducted a multidiscipline study with acupuncture and engineering science, for the component analysis and quantitatively heating measurement of fire needling process, and provides data support and suggestions for the clinical application of the fire needling therapy.

\section{Biography}

Professor Lin-Peng Wang, PhD supervisor, is the Director of Acupuncture Center in Beijing Hospital of Traditional Chinese Medicine affiliated to Capital Medical University, the Director of Acupuncture Education and Research Department of Traditional Chinese Medicine of Capital Medical University, a committee member of Neurology Department of Capital Medical University, the supervisor of the municipal government project of experience inheritance from TCM experts, and the academic leader of Key Discipline of Acupuncture and Moxibustion of Beijing and State Administration of Traditional Chinese Medicine.

Professor Ling-Peng Wang inherits the experience and academic theory of national TCM master Pu-Ren He and Le-Ting Wang, and has more than three decades of clinical experiences on acupuncture. He has hosted and completed more than ten national and municipal projects including National 973 Plan, National and Beijing Natural Science Foun- dation, project of State Administration of Traditional Chinese Medicine and the Beijing municipal science and technology plan. He has more than 90 academic and research publications, twelve papers of SCI and four books. Additionally, he is awarded by six ministerial and provincial prizes.

Professor Wang is specialized in treating migraine, stroke, neuromuscular disease, neuralgia and other pain diseases by acupuncture and moxibustion. He emphasizes the importance of a combination of Traditional Chinese Medicine and modern medicine in both clinic and research. His treatment theory and technique for migraine by acupuncture has gained good results and made him at the leading position in this field. His present work is to inherit and promote the use of traditional Chinese acupuncture in different diseases.

\author{
Korean Hand Therapy - An Overview and \\ New Aspect \\ Kyu Hyun Park, Tae Woo Yoo \\ Professor Emeritus and Director, Pusan National \\ University, Korea \\ E-Mail qhynbak@pusan.ac.kr
}

\section{Abstract}

Description: Korean Hand Therapy was discovered and developed by Dr. Yoo since 1971. According to KHT theory the hand is a microcosm of the human body and reflects the entire body. There are 14 micromeridians and 404 corresponding acupuncture points in the hands. This lecture introduces the concept of Correspondence Therapy which is essence of KHT. Correspondence Theory identifies the location of particular points and areas on the hands that correspond to the diseased part of the body. By stimulating the corresponding point on the hands, the ailment can be alleviated or cured. This therapy has proven to be very effective in alleviating the dysfunction of organs. In the view point of clinical practice, it is easy to learn KHT and to practice without side effects.

Objective: This is a basic course of Koryo Hand Acupuncture. In this theory, stimulating the reflex points on the hands can control all the functions of the internal organs. If there is pain or a disorder somewhere in the body, the corresponding points can be found on the hands by applying pressure to the hands. The corresponding points will show up as tender points on the hand. By stimulating the tender point on the hands, it will positively affect the corresponding part of the body. In KHT theory, the palm side of the hand represents the anterior side of the body, and the dorsal side of the hand represents the posterior side of the body. The second and fourth fingers correspond to the arms, and the first and last fingers to the legs. The point (K-A1) which is located $0.5 \mathrm{~cm}$ above the transverse crease of the wrist joint corresponds to the genital organs and the half way point (K-A8) of the vertical midline intersection with the transverse line on the palm (the center of the palm) corresponds to the navel. The center on 
the dorsum of the hands (K-B7) corresponds to the secondthird lumbar vertebra, and the K-B1 of the hands to the anus and the tip of the coccyx. By studying such a specific corresponding area, students will learn how to find and treat the reflex points.

Goal: This lecture will present the therapies stimulating the corresponding point on the hands by utilizing various KHT noninvasive stimulating instruments.

New Aspect: Gold meridians and acupuncture points on the body are developed from KHT micromeridians. Gold meridian is different from TCM meridian. We also need information to apply Gold meridian system without using inserting acupuncture needle on the body.

\section{Biography}

Kyu Hyun Park, MD, PhD is a Emeritus Professor of Neurology from March 2014. He is director of Center of Integrative Medicine, Pusan National University Hospital. He was professor of Neurology from 1989 to Feb. 2014 at Pusan National University, Busan, Korea. He is vice president of Korean Integrative Medicine Association. He has studied acupuncture since 1968, esp. Korean Hand Acupuncture since 1982. He has membership of National Academy of Medicine of Korea, Korean Association of Internal Medicine, Korean Neurological Association, Korean Geriatric and Gerontology, Korean Dementia Association, International Headache Society, Korean Hand Acupuncture.

\section{Acupuncture Regulation of Autonomic Functions Related to Locations of Points}

Xin-Yan Gao

Professor, China Academy of Chinese Medical Science, Institute of Acupuncture, China

E-Mail xinyangao2001@msn.com

\section{Abstract}

On the basis of recent series studies, we proposed that acupoint and the viscera innervated from the same spinal segment organize as a homotopic structure-function unit (dermatome) via sympathetic control. Contrarily, the acupoint innervated from other spinal segments organizes as a functional heterotopic unit via parasympathetic regulation. Both homotopic- and heterotopic-unit acupoints establish a homeostasis that somatic input regulates and balances visceral function. Homotopic acupoint is selected for parasympathomimetic hyperactivity and heterotopic acupoint for sympathomimetic hyperactivity, to rebuild systemic or organic homeostasis. For example, acupuncture at heterotopic acupoints (ST36) produce parasympathomimetic effect and acupuncture at homotpic acupoints (PC6) has sympathomimetic regulation on MAP and HR in anesthestized rats. Whereas for gastric motility, heterotopic acupoints (ST36) increases the peristalsis mimic parasym- pathomimetic regulation and homotopic acupint (ST25) restrains gastric contraction as the result of sympathomimetic regulation.

\section{Biography}

Gao Xinyan, $\mathrm{PhD}$, associate professor, head of the Department of Physiology. Dr. GAO has more than 40 publications in recent years. With the projects presided or participated, she has obtained 6 Awards of the Provincial and Ministerial-level. She has been invited as guest editor by five eCAM special issues and in the editorial board of Internet Journal of Alternative Medicine, Medical Acupuncture, Chinese Journal of Pain Medicine, Chinese Journal of Integrated Traditional and Western Medicine, et al. The research group first takes note of the auricle-vagus nerve correlation on the mechanism of auricular acupuncture and investigates the regulation of viscera autonomic nervous function. They constructed the theory of 'Unit' and 'Multiunit' acupoints for acupuncture points selection based on the body segments. The on-going studies also include: bidirectional regulation of functional intestinal disorders by acupuncture; biological mechanism for cross-talk of acupuncture and targeted organs; principles of moxibustion and the application, invention of moxibustion instruments; the endogenous pain modulation system and adenosine neurotransmitter system in acupuncture analgesia.

\section{Acupuncture Point Laterality Guang-Jun Wang \\ Associate Professor, Institute of Acupuncture and Moxibustion, China Academy of Chinese Medical Sciences, China \\ E-Mail tjuwgj@gmail.com}

\section{Abstract}

In the Yellow Emperor Neijing, there are such records as 'if someone has disease related with the left side, the treatment point is the right side, and vice versa', which emphasized that specific lateral side acupoint stimulation might lead to the theraputic advantages under the specific conditions. We summarized this difference effect resulted from the namesake acupoint stimulation as the acupoint laterality. Our studies indicated that acupuncture different side Neiguan (PC6) had the different effect on the heart rate variablility, which means that PC6 has the laterality. However, the difference can't be observed when the the same stimulation was applied to the either side Hegu samenake acupoint (LI4). Compared to the results from Neiguan, we consider that namesake acupoint laterality might related to the acupoint specificity. Although the evidence is not clear, our results indicated that in patients with hypertension, different side Quchi (LI11) stimulation can also produce different results, which might stand for some point of view that acupoint laterality might be associated with functional state of subjects. 
BIT's 2nd Annual World Congress of High-Tech Acupuncture and Integrative Medicine

\section{Biography}

Dr. Guang-Jun Wang, MD, Associate professor of Institute of acupuncture and Moxibustion, CACMS. Member of Beijing Society for Neuroscience, Director member of Beijing Society for Anatomical Sciences, Member of the China Association for Acupuncture and Moxibustion. He got his MD in Acupuncture and TCM in the Tianjin University of Traditional Chinese Medicine in 2007. Since then, he worked in the institute of Acupuncture and Moxibustion, CACMS. His research interests include the meridian explore, acupoint specificity and EEG analysis.

\section{Yellow Laser Acupuncture for Prevention and Early Intervention of Lifestyle-Related Diseases}

Daniela Litscher

Research Associate, Medical University of Graz, Austria E-Mail daniela.litscher@medunigraz.at

\section{Abstract}

Lifestyle-related diseases are a big problem worldwide. Not only the elderly population is affected by this group of diseases, also more and more young people are among the patients. Red, near infrared, green and violet lasers exist already, but - especially for diseases caused by the lifestyle the yellow laser represents an entirely new option in the field of laser acupuncture and could open up new ways of integrative therapy. In the present study, blood pressure (BP), heart rate variability (HRV), and temperature effects before, during, and after stimulation of different acupoints with yellow laser were investigated. The acupoints Baihui, Neiguan, Taichong and a placebo point were stimulated with a $589 \mathrm{~nm}$ ( $50 \mathrm{~mW}, 500 \mu \mathrm{m}$; $5 \mathrm{~min}$ ) yellow laser. Systolic BP decreased significantly after acupuncture, diastolic BP also decreased (n.s.), while HRV increased in both men and women. The skin temperature during stimulation decreased significantly in all measured points, but after the stimulation it increased again significantly. According to a questionnaire volunteers reported a significantly decreased level of stress after the stimulation. Significant positive effects on BP and well-being were found after yellow laser stimulation. The results are very promising and can be very important especially for the treatment of lifestyle-related diseases. Therefore further studies on the topic of yellow laser acupuncture will follow soon.

The study was supported by the Science Department of the City of Graz. A part of the travel costs related to this congress will be covered by the Office for Doctoral Studies of the Medical University of Graz.

\section{Biography}

Daniela Litscher studied Pharmacy at the Karl-Franzens University of Graz, Austria (from September 2008 to March 2013). Her diploma thesis dealt with 'Pharmacopuncture'. She received her Master degree in Pharmacy (MSc) on March 20th, 2013. She participated at several scientific conferences like the Laser Helsinki Congress (Helsinki, Finland; August 2008, 2010), Conferences of the Eurasia Pacific Uninet (Beijing/ Xian, China; May/June 2009, May 2011, May 2013), or Laser Congresses (Opatija, Croatia; June 2012, Toronto, Canada; November 2013). In September 2012, she visited the Korean Pharmacopuncture Institute in Seoul, Korea. In May 2014 she was a speaker at BIT's 1st Annual World Congress of HighTech Acupuncture \& Integrative Medicine 2014 in Nanjing, China. Currently, she works at the Medical University of Graz at the Research Unit for Complementary and Integrative Laser Medicine and the TCM Research Center Graz.

\section{How Much Is Enough? An Observational Study on Laser Ear Acupuncture in Mental Wellbeing}

Im Quah-Smith

Chief Executive Officer, Roseville Wellness Group, Australia

E-Mail quahsmith@gmail.com

\section{Abstract}

The Nogier Sign (NS) is a useful marker in the delivery of therapeutic laser in ear acupuncture. The duration of laser delivery or upload required according to the Nogier Sign (also known as the vasculo-autonomic signal or VAS) measures the optimal upload of laser energy required by the individual for each ear point at that session. With improvement of the condition being treated, the duration of laser delivery required is reduced. Twenty subjects were recruited from a primary care setting with presentations of emotional distress. Baseline, interim, end of treatment and post treatment emotional distress assessments were made using the DASS or Depression, Anxiety and Stress Scale. Up to six auriculotherapy points appropriate to mental health and wellbeing were stimulated with an infra-red therapeutic laser and the interval of radial pulse waveform change (VAS) was measured by palpation. The duration of laser energy upload for each ear point was recorded directly onto a specialisedcomputer programme controlled by the clinician. In the statistical analysis for each condition, the duration of laser upload at baseline and end of treatment was compared. Mean intervals decreased from baseline to end of treatment. Follow up analysis of the trend across treatment occasions was conducted. Both of these analyses were performed with repeated measures ANOVA. The highly significant results (changes) in laser upload duration per ear point will be reported at the congress.

\section{Biography}

Dr. Im Quah-Smith is an independent medical researcher and research associate at the Centre of Healthy Brain Aging, University of New South Wales. She is also a research asso- 
ciate at the Royal Women and Children's Hospital, Sydney, Australia, investigating the role of ear acupuncture in alleviating the pain and suffering of neonates with Neonatal Abstinence Syndrome. Her other projects in 2015 include the fMRI evidence for Battlefield Acupuncture and the Role of Acupuncture in Improving Human Resilience. Her recent body of work (publications) include the Clinical and Biological Evidence Base for Laser Acupuncture in Depression. She founded Roseville Wellness Group to create a space for healing patients and promote meditation, patient self-care and solutions for managing stress and chronic ill health. Dr. QuahSmith is also on the editorial committees of peer-reviewed journals: Medical Acupuncture and Acupuncture and Related Therapies. She is often guest editor and on the editorial committee of the National Faculty for Special Interests (NFSI) at the Royal Australian College of General Practitioners, Australia.

\section{Integrative Therapy for Increasing Cognitive Reserve}

\section{Weidong Pan}

Professor, Department of Neurology, Shuguang Hospital Affiliated to Shanghai University of Traditional Chinese Medicine, China

E-Mail panwd@medmail.com.cn

\section{Abstract}

Cognitive reserve is one kind of important integrative reservers of our body. The idea behind cognitive reserve is that the brain actively attempts to compensate for cognitive pathology. Some people are able to compensate better, for example, by using more efficient brain networks or alternate networks, and may be able to function more normally despite the pathology. People with more neurons might be able to lose more of them before showing a clinical deficit. The ability of cognitive reserve in traditional Chinese medicine (TCM) theory is the ability of kidney yin and yang, subjects with stronger kidney yin and yang have higher ability to resist the senility of cognitive. Many therapies of TCM including acupuncture could improve this ability of our brain. We demonstrated some success researches indicating TCM might be a effective method to protect our brain getting worse.

\section{Biography}

Prof. Weidong Pan is the associate director of the department of neurology, Shuguang hospital affiliated to Shanghai university of TCM. He has got bachelor degree of TCM in college of traditional Chinese medicine, Ningxia Medical University more than 20 years. He has studied clinical neurology in Tokyo University, Tokyo, Japan and got his doctoral degree and Post doctoral research process in graduate school of Tokyo University, Japan. Now he is the chairman committee of neuroendocrine committee of Shanghai integrative medicine association (since 2015), committee member of neurology committee of Shanghai integrative medicine association (since 2009), member of Chinese society of neuroscience and member of society of Chinese physician association. Editor-in-Chief of Integrative Medicine International (IMI), and he is editorial member of many international journals. He has published more than 60 publications in 5 years about traditional Chinese medicine and integrative medicine.

\section{The Biological and Clinical Evidence Base for Laser Acupuncture in Depression}

Im Quah-Smith

Research Associate, University of New South Wales, Australia

E-Mail quahsmith@gmail.com

\section{Abstract}

A pilot clinical study on using low level laser acupuncture in major depression revealed laser on acupuncture points LR8, CV14, LR14, HT7 significantly reduced the subjects' depression measure, the Beck Depression Inventory (BDI) Scores $(p<0.001)$. These acupuncture points were then biologically investigated using functional Magnetic Resonance Imaging (fMRI) with a 3 Tesla scanner. In study one, ten healthy subjects were scanned whilst undergoing randomized introduction of the above mentioned acupuncture points. The block design allowed a placebo-active sequence. A sham acupuncture point was introduced. In study two, ten subjects with major depression were also scanned using the above protocol. However, instead of the sham point, KI 3 important in the treatment of anxiety disorders was introduced into the fMRI acupuncture points protocol to test its relevance (if at all) in this sample. The results revealed there was more brain region activation and deactivation in the depressed subjects, in particular the posterior brain. Another interesting outcome was the modulation of the DMN or Default Mode Network important in the individual's ability to restore and maintain his or her sense of self (medial frontal gyrus). The sham point was not inactive. This important information has to be taken into consideration in future clinical acupuncture study designs. KI 3 was not significantly activated in this sample of major depressed subjects. Finally the results from the double blind randomized controlled pilot clinical study were replicated. The psychological measures were changed to include the clinician interviewed Hamilton Depression Scale (HAM-D) and the Quick Depression Inventory (QID). The laser acupuncture effect at end of treatment was highly significant $(\mathrm{p}<0.001)$ remained significant for 3 months post-laser ( $<<0.01)$. The only adverse effect was transient fatigue. Laser acupuncture is both time and cost effective and should be part of the armatorium of treatment protocols utilized by health authorities globally in their battle to contain the escalating costs of depression management today. 
Biography

Dr. Im Quah-Smith is an independent medical researcher and research associate at the Centre of Healthy Brain Aging, University of New South Wales. She is also a research associate at the Royal Women and Children's Hospital, Sydney, Australia, investigating the role of ear acupuncture in alleviating the pain and suffering of neonates with Neonatal Abstinence Syndrome. Her other projects in 2015 include the fMRI evidence for Battlefield Acupuncture and the Role of Acupuncture in Improving Human Resilience. Her recent work has included strategies to individualise the dosage of acupuncture for optimal outcomes. She founded Roseville Wellness Group (RWG) to create a space for healing patients and promote meditation, patient self-care and solutions for managing stress and chronic ill health. Dr. Quah-Smith is also on the editorial committees of peer-reviewed journals: Medical Acupuncture and Acupuncture and Related Therapies. She is often guest editor and on the editorial committee of the National Faculty for Special Interests (NFSI) at the Royal Australian College of General Practitioners, Australia.

\section{Successful Modulation of Complex Regional Pain Syndrome Type 1 by Injection of Acupuncture Points with Placental Extract: A Case Report}

Tae Hwan Cho ${ }^{1}$, Kyeong Mee Park ${ }^{2}$

${ }^{1}$ Cho Orthopaedic Clinic, ${ }^{2}$ Inno Oriental Clinic, Korea

E-Mailkmpark02@empas.com

\section{Abstract}

The case of a female patient who developed complex regional pain syndrome (CRPS) in the left upper limb after a traumatic injury on the distal part of the left forearm is reported. The pain was immediate and resistant to both oral analgesics and continued transcutaneous electrical nerve stimulation. Five months after the injury, the patient presented to our clinic with severe pain, swelling, redness, cold sensation of left hand and even loss of function from the left hand up to the left shoulder. Acupuncture points LI5, LU2, SI10, HT1, GB21 and SI11, which are localized in the joints or the muscles responsible for the movement of the left upper limb, were selected for application of placental extract. Injection of placental extract into the acupuncture points resulted in dramatic pain relief, swelling remission, motor recovery, temperature normalization and disappearance of redness in this CRPS type 1 patient.

\section{Biography}

1990 2000: Research scientist in the department of pharmacology of Korea Ginseng \& Tobacco Research Institute.

2000 2002: Senior research scientist in the department of pharmacology of Korea Ginseng \& Tobacco Research Institute.
2002 2003: Senior research scientist in the central research institute of KT\&G.

2011 present: Oriental medical doctor in Inno Oriental Clinic.

\section{Effect of Perioperative Electroacupuncture on Postoperative Analgesia in Prostatectomy at the General Hospital of Thessaloniki 'G.Gennimatas'}

Vagia Ntritsou

Consultant Anesthesiologist, General Hospital of

Thessaloniki 'G.Gennimatas', Greece

E-Mail vaya_ntr@yahoo.gr

\section{Abstract}

The analgesic effect of electroacupuncture as perioperative adjunctive therapy added to a multimodal systemic analgesic strategy with tramadol and ketamine for postoperative pain, opioid-related side effects and patient satisfaction was studied. 75 patients undergoing radical prostatectomy under general anaesthesia were randomly assigned to two groups: 'EA' ( $\mathrm{n}=35$ ) Tramadol+Ketamine+Electroacupuncture and 'Control' ( $\mathrm{n}=35$ ) Tramadol+Ketamine. Electroacupuncture applied at $100 \mathrm{~Hz}$ frequency at LI4 bilaterally for $30 \mathrm{~min}$ during the closure of the abdominal walls and at $4 \mathrm{~Hz}$ at ST36 and LI4 bilaterally just after extubation for $30 \mathrm{~min}$. All patients were administered a continuous infusion of $0.15 \mathrm{mg} / \mathrm{kg}$ tramadol and ketamine iv. Postoperative pain was evaluated by recording patients' pain using Numerical Rating Scale (NRS), McGill Scale (SF_MPQ), mechanical pain thresholds after algometer application close to the wound, cortisol measurements, rescue analgesia, Spielberger State Trait Anxiety Inventory (STAI Y-6 item), patient satisfaction and side effects. Pain scores on the NRS and SF_MPQ were significantly lower and electronic pressure algometer measurements were significantly higher in the 'EA' group compared to the 'Control' group $(\mathrm{p}<0.001)$ at all assessments. Significant decrease in rescue analgesia was observed at $45 \mathrm{~min}$ in the 'EA' group ( $p<0.001)$ as well as in cortisol levels in the 'EA' group $(\mathrm{p}<0.05)$. Patients were significantly satisfied with analgesia, especially in the 'EA' group $(p<0.01)$. Significant delays in the beginning of bowel movements were observed in the 'Control' group at $45 \mathrm{~min}(\mathrm{p}<0.001)$ and $2 \mathrm{~h}(\mathrm{p}<0.05)$. Adding electroacupuncture application perioperatively would be an interesting option as a part of a multimodal analgesic strategy as it decreased postoperative pain and opioids related adverse effects and improved patients' wellbeing.

\section{Biography}

Dr. Ntritsou is a consultant anesthesiologist in General Hospital of Thessaloniki 'G. Gennimatas', Doctor of medical sciences, several lectures in her country, 9 scientific publications, partly on postoperative analgesia and member of the editorial board of Greek scientific journals Themata of Anaes- 
thesiology and Intensive Care. Dr. Ntritsou's special interests are postoperative analgesia and her $\mathrm{PhD}$ was on the effect of perioperative electroacupuncture as an adjunctive therapy on postoperative analgesia with continuous infusion of tramadol and ketamine in prostatectomy which was published in Acup Med 2014;32:215-222.

\section{Electro-Acupuncture Therapy for Ophthalmoplegia: A Case Series}

\section{Zhidan Liu}

Baoshan Hospital of Integrated Traditional Chinese Medicine and Western Medicine, Shanghai University of Traditional Chinese Medicine, Shanghai, China E-Mail liu54643@163.com

\section{Abstract}

Baoshan Hospital of Integrated Traditional Chinese Medicine (TCM) and Western Medicine, Shanghai, also called Shuguang Hospital Affiliated to Shanghai University of TCM Baoshan branch, which is the integrative medical center of Baoshan district, and the teaching hospital of Shanghai University of TCM as well as Nanjing University of TCM. The ophthalmology department of is one of the distinguished discipline of ophthalmology and TCM in Shanghai. It is the largest medical center of Baoshan District.

Ophthalmoplegia is a common clinical problem, the most reason is muscle paralysis caused by nerve palsy, such as oculomotor or abducens nerve palsy, which cause inconsistent movement of related muscles, resulting in diplopia, headache and other symptoms. In China, western medicine, TCM and acupuncture are used to treat related symptoms reported in literatures, but the selected treatment methods varied widely. We are under groping the therapy of a group of selected acupuncture points based on experiences from veteran practitioner of TCM, which we shared in the text. We will try to improve the therapeutic manipulations, but randomized controlled studies are needed to conclude the definite efficacy.

\section{Biography}

Dr. Liu is a licensed acupuncture practitioner and scientific research fellow of 10 years in Baoshan Hospital of Integrated Traditional Chinese Medicine (TCM) and Western Medicine, Shanghai. He graduated from Hubei University of TCM and Shanghai University of TCM, under the direction of Prof. Dr. Pei Jian, the director of Acupuncture and moxibustion department, Longhua Hospital, Shanghai University of TCM. And now he is a PhD student in Shanghai University of TCM, under the direction of Prof. Dr. Shen Weidong, the director of Acupuncture and moxibustion department, shuguang Hospital, Shanghai University of TCM.

Dr. Liu's special interests are regulating mechanism of acupuncture on immune function and neuromuscular disease treatment. His researches have been supported by National
Science Fundation, Shanghai Municipal of Health and Family Planning Commission and Science and Technology Development Fund of Baoshan District, Shanghai. He has published about 20 scientific papers, one of which with SCI journals.

\section{Substance P Is One of Crucial Elements in Activating Acupuncture Effect}

\author{
Bo Chen \\ Tianjin University of Traditional Chinese Medicine, \\ China \\ E-Mail tjutcmchenbo@163.com
}

\section{Abstract}

Although acupuncture has been used throughout the world, its mechanism is still in exploring. Acupoints are the main sites to receive the stimulation of acupuncture, so changes occur at the microenvironment of acupoints are the initial reaction to insertion of the needle. According to the neurophysiology, physical stimulation, as well as acupuncture, can cause the excitement of local afferent fibers, activation of relevant cells (e.g. mast cells) and release of various chemical substances. Our research team focuses on the interaction of the above-mentioned three factors of the acupoints, and their relationship with the acupuncture effects. In our recent experiment, we used $2 \mathrm{U} / \mathrm{kg}$ pituitrin solution to establish the bradycardia model of rabbits, observed the effect of $2 \mathrm{~Hz}$ electroacupuncture (EA) applied to Neiguan (PC6) and the concentration of Substance P (SP) at PC6 in different time periods by radioimmunoassay. Then we injected $1 \mathrm{~g} / \mathrm{L} \mathrm{NK}-1$ receptor (SP agonist), $1 \mathrm{~g} / \mathrm{L}$ spantide (SP receptor antagonists) and $0.01 \mathrm{M}$ phosphate buffer solution $50 \mu$ linto PC6 respectively to observe the changes of therapeutic effect of EA. The results showed that EA can raise the SP concentration at PC6 of the bradycardia rabbits. The therapeutic effects of each group were as follows: agonist group > EA group (PBS group) $>$ model group $>$ antagonists group; no statistical difference was showed between EA group and PBS group. There existed a positive correlation between SP concentration at PC6 and the heart rate recovery effect. Our research indicates that local SP may be one of crucial elements in activating acupuncture effect.

\section{Biography}

Chen Bo, a doctor of acupuncture and moxibustion, engaged in the study of acupuncture clinical design, acupuncture mechanism, Acupuncture and moxibustion standardization and blood-letting and cupping. He has published over 50 theses in national core periodicals and 9 articles in non-Chinese language periodicals (4 SCI papers, 1 Japanese paper) and invited as the reviewers of PloS ONE, Patient Preference and Adherence, Medical Hypotheses, etc. He has published Teachers' health instruction manual and participated in a number of state-level project researches, such as Study on the law of acupoints compatibility effect and 
Neurobiological mechanisms (The national basic research program of China (973 program)), Study on the regulating effect and mechanism of acupuncture on Neuro-EndocrineImmune network (National Natural Science Foundation of China), Study on the initial Dynamic regulatory mechanism ofacupuncture effect initiation (National Natural Science Foundation of China), Study on the promoter of acupuncture effects: functions and mechanisms of purinergic signaling in the initial regulation of acupuncture effects (National Natural Science Foundation of China), Primary research on nerve-immune regulation initating mediated by mast cellderived exosome through needling acupoints (National Natural Science Foundation of China). He has finished one part of the experiment of Study on the initial Dynamic regulatory mechanism ofacupuncture effect initiation and is studying the research on clinical effect and some mechanism of prevention and treatment of nausea and vomiting after chemotherapy by acupuncture.

\section{Effects of GUASHA by Improving Heart Rate Variability and Reducing Perceived Exertion in Male Weightlifter}

Xingze Wang ${ }^{1}$, Wichai Eungpinichpong ${ }^{2}$, Jinsheng Yang ${ }^{3}$

${ }^{1}$ Faculty of Physical Education Gannan Normal University, China; ${ }^{2}$ Research Center in Back, Neck, Other Joint Pain and Human Performance, Khon Kaen University, Thailand; ${ }^{3}$ Institute of Acupuncture and Moxibustion, China Academy of Chinese Medical Sciences, China E-Mail wxz1266@126.com

Background: GUASHA is a traditional Chinese therapy in which the subject is scraped repeatedly by using a blunt spoon-like object on certain areas of the body. The current study aims to evaluate the effects of 8-weeks of GUASHA on the rate of perceived exertion (RPE), and heart rate variability (HRV) by comparing with the sham scraping in weightlifters under normal weightlifting training.

Methods: Randomized controlled trial was chosen in this study, 30 subjects who met the inclusion criteria was randomly allocated to GUASHA group or sham scraping group using stratified random allocation by using athletic level. They underwent 16 sessions of treatment during normal weightlifting training for 8 weeks. To compare the effects of GUASHA therapy after the treatment, RPE of snatch, clean and jerk (85\% 1RM), HRV were measured.

Results: RPE of snatch, clean and jerk were significantly reduced after GUASHA in GG and SS, whereas, there was a significant difference in LF and LF/HF $(\mathrm{P}<0.05)$, LF decreased, and the ratios of LF/HF decreased.

Conclusions: GUASHA therapy could reduce perceived exertion, and increase HRV response, it is recommended that it should be considered as an alternative treatment for sport recovery.

Keywords: RPE, HRV, GUASHA therapy, scraping, normal weightlifting training.

\section{Biography}

Dr. Xingze Wang is a teacher in Gannan Normal University now, associate professor, working in the Institute of Physical Education. He exercised weightlifting and achieved the grade one player at 1995, learned theory pedagogy and training during college from 1995 to 1997 . He was a teacher of physical education in middle school from 1997 to 2001, coached the weightlifting team in the institute of physical education in Shanghai from 2001 to 2004, and doing PhD in Khon Kaen University, Thailand, Human Movement Sciences, Physical Therapy from 2011 to 2014, to now as an associate professor in Gannan Normal University. And he has published 2 pieces papers about GUASHA therapy in the journal of traditional Chinese medicine, and Evidence-based Complementary and Alternative Medicine. 


\section{Selected Abstracts - Poster Presentations}

\section{The Influence of Tuina in Reducing Crying and Fussing in Infants with Infantile Colic - A Clinical Research Protocol}

Sara Duarte ${ }^{1}$, Maria João Santos ${ }^{1}$, Nuno Correia $^{1,2}$, Jorge Machado ${ }^{1,3}$, Henry Johannes Greten ${ }^{1,4}$

${ }^{1} \mathrm{TCM}$ Master Programme, Abel Salazar Institute for Biomedical Sciences, University of Porto, Porto, Portugal; ${ }^{2}$ Acupncture Clinic, Alfena Private Hospital, Porto, Portugal; ${ }^{3}$ Labiomep-Porto Biomechanics Laboratory - University of Porto, Portugal; ${ }^{4}$ Heidelberg School of Chinese Medicine, Heidelberg, Germany

Background: In Western societies, incident rates of infantile colic go up to $25 \%$. Infants who cry persistently are common concerns for parents and, more rarely, the crying may lead a parent to smother, hit or shake an infant, sometimes resulting in infant brain damage or even death. Also in Western medicine current medical treatments are inefficient (simethicone) or still under research (probiotics and prebiotics).

Objective: The purpose of this study is to assess the influence of Tuina in reducing duration and intensity of crying in infants related to infantile colic.

Methodology: This is a prospective, randomised, controlled and single-blinded study.

Inclusion Criteria: Infants with 2-8 weeks of age; healthy; born after gestational week 37 ; with a weight $\geq 2500$ g; fulfilling the modified Wessel criteria for infantile colic (crying/fussing for at least 3 hours a day (cumulative), occurring 3 days or more in the same week).

Exclusion Criteria: Poor compliance when parents do not fill de crying and fussing diary.

Intervention: The experimental group will be subject to Tuina, whereas the control group will have no therapy.

Measurements: Parents report the infants' crying, fussing and colicky crying in a diary before the beginning of the study and then during the study.

Outcomes: Total duration of fussing, crying and colicky crying in a day during the intervention period as reported by the parents in the diary.

Expected Results: This preliminary study may show a reduction on the total daily duration of fussing, crying and colicky crying is expected to be shorter in infants of the intervention group, compared to infants included in the control group.

Keywords: Tuina, crying infants, infantile colic.

\section{Immediate Effects of the S34 Puncture Point on the Degree of Flexion-Extension Amplitudes of the Lumbar Spine in Healthy Individuals}

Estrela Ferreira ${ }^{1}$, Maria João Santos ${ }^{1}$, Nuno Correia ${ }^{1,2}$, Jorge Machado ${ }^{1,3}$, Henry Johannes Greten ${ }^{1,4}$

${ }^{1}$ TCM Master Programme, Abel Salazar Institute for Biomedical Sciences, University of Porto, Portugal; ${ }^{2}$ Acupncture Clinic, Alfena Private Hospital, Porto, Portugal; ${ }^{3}$ Labiomep-Porto Biomechanics Laboratory - University of Porto, Portugal; ${ }^{4}$ Heidelberg School of Chinese Medicine, Heidelberg, Germany

Background: Diseases related to spine have increased significantly due to inactivity and the adoption of incorrect postures. In addition to body support, the vertebral column also offers the necessary flexibility to the movement of the torso, playing an important role in the raised posture, locomotion and balance.

Objectives: To evaluate the effects of puncture S34 point on the scale of the movements of flexion-extension of the lumbar spine in healthy individuals.

Methodology: A clinical pre-study with a randomized, controlled and single-blinded design. Sample: 30 volunteers $(\mathrm{N}=30)$ : 15 individuals in the placebo group (needling on the extra point between S34 and F32) and 15 subjects allocated to the experimental group (puncture on S34).

Inclusion Criteria: Healthy individuals between 25-40 years-old.

Exclusion Criteria: Patients with sciatica, lumbago, disorders within the sacral-iliac or orthopedic prosthesis (knee or pelvic).

Intervention: A single manual acupuncture session will be performed during 30 minutes.

Outcomes: (1) Pain or discomfort and (2) flexionextension and right-left amplitudes of the back.

Measurements: Pain or discomfort will be assessed by visual analogue scale (VSA) and flexion-extension and rightleft amplitudes will be measured by an electronic goniometer fixed in space between L4-L5 and L1-L2. This device will be connected to a computer program (Biopac Student Lab.), recording the angles resulting from the maximum extension movement, maximum bending and maximum right and left movements of the lower back.

After baseline (T0) measurements, intervention will take place following the technique of 'Leopard-spot' needling 
(inserting and removing 4 times an insulin needle on the acupoints).

After 5 minutes of rest, VSA and inclinometry will be assessed (T1).

Expected Results: This preliminary study is intended to demonstrate the immediate effects on the extent of flexionextension and lateral movements of the lumbar spine in healthy individuals through the puncture of the S34. By showing improvements, a specific effect of needling S34 may be objectively demonstrated.

Keywords: Flexion-extension amplitude, lumbur spine, $\mathrm{S}$ 36 (St 36).

\section{The Effect of Qigong in Attention Deficit Hyperactivity Disorder: A Clinical Research Protocol}

\author{
Marta Correia ${ }^{1}$, Maria João Santos ${ }^{1}$, Nuno Correia ${ }^{1,2}$, \\ Jorge Machado ${ }^{1,3}$, Henry Johannes Greten ${ }^{1,4}$ \\ ${ }^{1}$ TCM Master Programme, Abel Salazar Institute \\ for Biomedical Sciences, University of Porto, Porto, \\ Portugal; ${ }^{2}$ Acupncture Clinic, Alfena Private Hospital, \\ Porto, Portugal; ${ }^{3}$ Labiomep-Porto Biomechanics \\ Laboratory - University of Porto, Portugal; ${ }^{4}$ Heidelberg \\ School of Chinese Medicine, Heidelberg, Germany
}

Introduction: Attention Deficit Hyperactivity Disorder (ADHD) is one of the most common chronic disorders. Frequently diagnosed during childhood, it may persist through adolescence and even show in adults. It may impair school performance, academic education and the professional life of patients with consequences for the whole social prognosis.

Besides cognitive-behavioural therapy, combined treatment with stimulant medication may be recommended. However, success of this treatment is insufficient and sideeffects may induce compliance problems. Consequently, there is a high demand for more therapeutic options.

Objectives: The purpose of this study is to assess the effect of 'White ball' Qigong on attention in Children with Attention Deficit Hyperactivity Disorder (ADHD) as measured by the D2 test.

\section{Methodology:}

Study Design: Prospective Randomized 3-Armed Placebo Controlled Single Blinded Trial in parallel group design.

The effect of Qigong on ADHD on three groups of children will be observed $(n=30$, each). The verum group will receive training in the verum exercises, the control group will receive training in special placebo Qigong exercises, a third group will not receive more specific treatment (waiting list). Exercises will be repeated daily at home, during four weeks and documented in a diary.

Inclusion Criteria: Children from 12 to 14 years, with ADHD diagnosis by an independent psychiatrist and pediatrician, stable pharmacological treatment for more than
3 months, informed consent signed by the parents and voluntary participation of the child.

Exclusion Criteria: Other mental or learning disorder, intelligence defect, relevant handicaps as an obstacle to the exercises.

Intervention: Verum and placebo qigong as described in detail previously. Participants will receive two ten-minute sessions per week by a certified trainer for four weeks and practice the exercises once daily at home.

Main Parameters: The intervention impact will be assessed by:

(1) A neuropsychological assessment of selective and sustained attention (D2 - Test of Attention);

(2) A psychological assesssment of ADHD (Conner's Parent Rating Scales),

(3) Physiological parameters, as heart rate (HR) and heart rate variability (HRV).

Theses assessments will be taking place at T0 (baseline), T1 (after 14 days), T2 (after 4 weeks) 1 month.

Expected Results: After four weeks of intervention, this study may reveal an increase of selective attention, on the vegetative stabilization as measured by HRV and a decrease of values in Conner's Rating Scales. Scales.

Keywords: White Ball Qigong, ADHD, Conner's Rating

\section{Acute Effects of Cupping Therapy, as a Therapeutic Modality in Traditional Chinese Medicine, on the Treatment of Temporo- Mandibular Joint Dysfunction - A Pilot Study}

Anabela Manso Silva ${ }^{1}$, Maria João Santos ${ }^{1}$, Jorge Machado ${ }^{1,2}$, Vânia Barros ${ }^{1,3}$, Nuno Correia ${ }^{1,3}$, Henry Johannes Greten ${ }^{1,4}$

${ }^{1}$ TCM Master Programme, Abel Salazar Institute for Biomedical Sciences, University of Porto, Porto, Portugal; ' Labiomep-Porto Biomechanics Laboratory University of Porto, Portugal; ${ }^{3}$ Acupncture Clinic, Alfena Private Hospital, Porto, Portugal; ${ }^{4}$ Heidelberg School of Chinese Medicine, Heidelberg, Germany

Background: Facial pain commonly results from Temporomandibular Joint (TMJ) dysfunction. This is a common problem often affecting people from 20 to 40 years of age. Around $33 \%$ of the population has at least one temporomandibular symptom and $3.6 \%$ to $7 \%$ of the population has TMJ disorder with sufficient severity to cause them to seek treatment. However, many of these patients are often resistant to conventional therapy.

Cupping therapy, as a technique of Traditional Chinese Medicine, may be effective in pain reduction.

Objectives: To assess the effects of cupping therapy in pain and jaw movement in patients with temporomandibular joint dysfunction. 
Methodology:

Study Design: A single-blinded, randomized, controlled study design.

Sample: 50 individuals.

Inclusion Criteria: Subjects over 18 years-old with a diagnose of temporomandibular joint dysfunction by a physician and a Stage III of ALT (Heidelberg Model).

Exclusion Criteria: Subjects with no limitation of the dental occlusion and subjects with associated disorders (systemic disorders).

Intervention: The participants oral cavity will be examined according to a previous establish protocol and the participants will be evaluated according to the Heidelberg Model of TCM. This include assessing the pain by pressure algometry and the opening movement of the jaw by a millimeter scale. Then, the participants will be divided into two groups that will receive a moving cupping treatment: experimental group - cupping on GB21, St13 and TB5 zone; control group - cupping on the vesicle conduit. After a rest period (5 min), a reevaluation will be performed.

Main Parameters: Objective measurements: pain, measured with pressure algometry, and dental occlusion, measured with TheraBite ${ }^{\circledR}$ (a specific scale for TM) movement measurement). Subjective measurements: the Craniomandibular Index.

Expected Results: This preliminary study may reveal a reduction on pain and increased in mouth opening by using cupping therapy in TMJ patients.

Keywords: Temporo-mandibular joint dysfunction, craniomandibular index, cupping.

\section{The Influence of Acupuncture and Phytotherapy in the Treatment of Chronic Inflammatory Diseases - A Feasibility Study}

Maria Helena Rocha da Silva ${ }^{1}$, Maria João Santos ${ }^{1}$, Nuno Correia ${ }^{1,2}$, Jorge Machado ${ }^{1,3}$, Henry Johannes Greten ${ }^{1,4}$

${ }^{1}$ TCM Master Programme, Abel Salazar Institute for Biomedical Sciences, University of Porto, Porto, Portugal; ${ }^{2}$ Acupncture Clinic, Alfena Private Hospital, Porto, Portugal; ${ }^{3}$ Labiomep-Porto Biomechanics Laboratory - University of Porto, Portugal; ${ }^{4}$ Heidelberg School of Chinese Medicine, Heidelberg, Germany

Background: Chronic inflammation is a manifestation of many health problems which include: arthritis, atherosclerosis, Alzheimer's disease, inflammatory bowel syndrome, infection and injury among others.

Chronic inflammation may be the engine that drives many of the most feared illnesses of middle and old age. The treatment methods generally include anti-inflammatory drugs (NSAIDs) such as aspirin, acetaminophen and ibuprofen, or corticosteroids. In any cases, its administration is associated with numerous side effects.
Acupuncture and phytotherapy can improve the patient's functional capacity leading to a reduction of the use of conventional drugs.

Objective: To evaluate the a general anti-inflammatory effect of acupuncture \& phytotherapy combined with conventional therapeutic.

Methodology: Sixty cases of chronic inflammation disease will be randomly divided into an acupuncture \& phytotherapy \& conventional experimental group and a control group with only conventional therapeutic. Acupuncture and phytotherapy will be selected according to a TCM diagnosis for patients' main complaint.

Inclusion Criteria: Male or female adults who suffering from chronic inflammation disease and those who signed the informed consent.

Intervention: 6 to 10 weeks of treatment.

Outcomes: The treatment impact will be assessed by C-reactive protein determination, an acute phase reactant and marker of inflammation.

Expected Results: The study may show a significant reduction on the inflammation marker by combined therapy of acupuncture + phytotherapy + conventional in comparison to standard conventional drug treatments.

Keywords: Chronic inflammatoriy diseases, inflammation marker.

\section{Vegetative Functions Induced by Music Following TCM Principles - A Preliminary Study \\ Sofia Sousa ${ }^{1}$, Cláudia Sousa ${ }^{1}$, Maria João Santos ${ }^{1}$, Carla Paiva ${ }^{1,2}$, Jorge Machado ${ }^{1,3}$, Henry Johannes Greten ${ }^{1,4}$ \\ ${ }^{1}$ TCM Master Programme, Abel Salazar Institute for Biomedical Sciences, University of Porto, Porto, Portugal; ${ }^{2}$ University of Minho, Braga, Portugal; ${ }^{3}$ Labiomep-Porto Biomechanics Laboratory - University of Porto, Portugal; ${ }^{4}$ Heidelberg School of Chinese Medicine, Heidelberg, Germany}

Introduction: Music is an emotional language and affects people through the central and the autonomous nervous systems (ANS). People give physiological responses when listening to music. The ANS is believed to be the way emotions induced by Music carry most of its therapeutic effects [Ellis and Thayer, 2010; Hodges, 2010]. Traditional Chinese Medicine (TCM) is a system of findings and sensations designed to establish the functional vegetative state of the body [Greten, 2006]. In TCM there is a teaching of healing sounds and of 'resonance'. The Heidelberg model highlights the role of emotionality in vegetative regulation and, therefore, can be a major contributor in understanding the effects of Music as emotion inductor.

Objectives: To study Music in terms of vegetative functions and emotionality. To allocate some excerpts of musical 
pieces to the phases, according to the physiological effects observed in healthy volunteers.

Methods and Study Design: Healthy volunteers will be evaluated before, during and after listening to music, through heart rate variability (HRV).

$\mathrm{HRV}$ is a validated measure of autonomic (dys)function and it is a measure of the continuous interplay between sympathetic and parasympathetic influences on heart rate that yields information about autonomic flexibility and thereby represents the capacity for regulated emotional responding.

Electromyography will be used to objectify the latency of muscle reaction.

Expected Results: With this experimental protocol, we expect to document a group of easily accessed musical pieces that may have different effects, according to the so-called phases of TCM. This study should be an initial step to prescribe music with defined proposes.

Keywords: TCM and music, phases of TCM, emotion inductor.

\section{Qigong: Improvement on Quality of Life, Motivation, Self-Regulation and Anthropometric Parameters on Overweight and Obese Individuals - A Pilot Clinical Study}

Catia Andreia Ferreira ${ }^{1}$, Maria João Santos ${ }^{1}$, Jorge Machado ${ }^{1,2}$, Henry Johannes Greten ${ }^{1,3}$

${ }^{1}$ TCM Master Programme, Abel Salazar Institute for Biomedical Sciences, University of Porto, Porto, Portugal; ' ${ }^{2}$ Labiomep-Porto Biomechanics Laboratory University of Porto, Portugal; ${ }^{3}$ Heidelberg School of Chinese Medicine, Heidelberg, Germany

Introduction: Obesity is one of the leading health risk factors worldwide. Qigong is a traditional vegetative biofeedback therapy that combines postures, movements with breath control and a special mental state of 'awareness'. One of the major obstacles on a program of weight management is the anxiety that naturally results from the 'rules' of eating healthy food. Qigong seems to have a really good effect on the stress reduction state and anxiety control.

Objectives: To study the effect of Qigong on (1) the treatment of reestablishing the harmony (qi flow), (2) improvement of the maintenance of motivation to 'stay ' on a dietetic program and self-regulation, (3) improvement of quality of life, (4) improvement of anthropometric parameters, and (5) improvement on stress and anxiety levels.

Methodology: A minimum of 45 individuals with obesity (class I and II) will be randomly divided into two experimental groups: (A1) $n=15$ (conventional diet for weight loss + gi gong) and (A2), $\mathrm{n}=15$ (conventional diet for weight loss + placebo gi gong); and a control group, $\mathrm{n}=15$ (conventional diet for weight loss only).
Intervention: Qigong: $1^{\text {st }}$ session - explanation of the study and the intervention/exercises demonstration for daily application of qi gong at home. Reinforcement sessions - once a week (on the training center where the patients were recruited) for practice, for taking doubts, for reinforce motivation and for more explanations of the exercises. Dietary advices: Conventional therapy - dietetic plan for weight loss established by a the Dietitian.

Outcomes: Quantitative measures: Body weight/perimeters (waist and hip)/BMI (body mass index), \% body fat; Qualitative measures: QoL questionnaires validated for the Portuguese population (SF8). Questionnaires on motivation and self-regulation: TREATMENT SELF-REGULATION QUESTIONNAIRE (TSRQ); THE PERCEIVED COMPETENCE SCALE (PCS).

Expected Results: This preliminary study may demonstrate the benefit of Qigong as a complementary therapy to include in a weight management program.

Keywords: Obesity, QoL, TSRQ, PCS.

\section{The Influence of Acupuncture in Balance After Maximum Exercise in Kickboxing Players - An Intervention Study}

\author{
Sara Moreira ${ }^{1}$, Maria João Santos ${ }^{1}$, Jorge Machado ${ }^{1,2}$, \\ Henry Johannes Greten ${ }^{1,3}$ \\ ${ }^{1} \mathrm{TCM}$ Master Programme, Abel Salazar Institute \\ for Biomedical Sciences, University of Porto, Porto, \\ Portugal; '2Labiomep-Porto Biomechanics Laboratory - \\ University of Porto, Portugal; ${ }^{3}$ Heidelberg School of \\ Chinese Medicine, Heidelberg, Germany
}

Background: Kickboxing is a martial art that combines kicks and punches which involves basic skills and combination exercises. The training effects on balance and mobility are considered by researchers as an important therapeutic measure to improve balance for individuals with multiple sclerosis. However, there are no other published studies focusing on examination of the effect of balance in kickboxing among healthy athletes. Thus, in kickboxing training, balanced movements are stronger, more powerful, faster, and take less energy. They are considered by researchers as an important measure for injury prevention.

Objectives: The aim of this study is to describe if there is an immediate effect of acupuncture in the balance level after a maximum in kickboxing players.

Methodology:

Study Design: An intervention clinical pre-study, with a randomized, controlled, single-blinded design. One verumacupuncture group, $\mathrm{n}=20$, and one control group (false acupuncture), $\mathrm{n}=20$.

Sample: A convenience sample of Kickboxing Players $(n=40)$. 
Inclusion Criteria: The athlete must have basic technical knowledge of the techniques to be used (white belt level) and have steadily trained kickboxing for at least 6 month prior to the test.

Exclusion Criteria: Injury, consumption of performance enhancing supplements in the week prior to the test.

Intervention: Balance will be assessed on the intervention group of Kickboxing players before and after a series of techniques (punches and kicks). Then, the athletes will be subjected to a high intensity training. After this step, the intervention group will be subject to needling on the S34. Finally, after acupuncture, balance will be measured again before and after the same series of techniques (punches and kicks). The control group will be subject to needling of a nonacupoint.

Measurements: A balance measurement to evaluate the protocol before and immediately after a short high intensity training.

Expected Results: Needling of S34 may improve balance in kickboxing players after maximum exercise. The study may in addition prove a specific effect of S34.

Keywords: Kickboxing, S34 (St 34), balance.

\section{Acute Effect of Body Acupuncture on Postural Stability in Healthy Subjects}

Tânia Fernandes ${ }^{1}$, Ana Anjos ${ }^{1}$, Luis Carvalho ${ }^{1}$ Jorge Machado ${ }^{1,2}$, Henry Johannes Greten ${ }^{1,3}$

${ }^{1}$ TCM Master Programme, Abel Salazar Institute for Biomedical Sciences, University of Porto, Porto, Portugal; ${ }^{2}$ Labiomep-Porto Biomechanics Laboratory University of Porto, Portugal; ${ }^{3}$ Heidelberg School of Chinese Medicine, Heidelberg, Germany

Introduction: The humans are bipeds and locomote over the ground with one foot in contact (walking), no feet in contact (running), or both feet in contact (standing) creating a major challenge to our balance control system. This feature plays an important role in our day to day activities. Therefore abnormality in postural stability may lead to serious consequences adversely affecting the normal life.

In Traditional Chinese Medicine (TCM), the human body has a symmetrical structure and maintains a physiological balance through the harmony of Yin and Yang.

As a system of findings and sensations designed to establish the functional vegetative state of the body, TCM as the potential to improve postural control and its influence in human being's health.

Objectives: The purpose of this experimental study is to evaluate in a healthy population, physically active, the effectiveness of body acupuncture in postural stability using a stabilometer.

Methods and Study Design: The volunteers' postural stability will be evaluated with stabilometry before and after acupuncture. This will be a blinded-control assay and two groups will be formed: one group with acupuncture in acupoints S34 selected to improve postural stability; and another with sham acupuncture, in extra point between S34 and F32 with no expected therapeutical effect.

Expected Results: With this experimental protocol, according to the bibliography, it is expected that acupuncture improve posture stability as objectively assessed by a stabilometer.

Keywords: Postural stability, stabilometry.

\section{Effects of Acupuncture on Gait of Patients with Multiple Sclerosis}

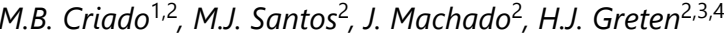 \\ ${ }^{1}$ CESPU-Instituto Politécnico de Saúde do Norte, \\ Gandra-Paredes, Portugal; ${ }^{2}$ TCM Master Programme \\ Abel Salazar Institute for Biomedical Sciences, \\ University of Porto, Porto, Portugal; ${ }^{3}$ Labiomep- \\ Porto Biomechanics Laboratory - University of Porto, \\ Portugal; ${ }^{4}$ Heidelberg School of Chinese Medicine, \\ Heidelberg, Germany
}

Introduction: Multiple sclerosis (MS) is considered a disease of the central nervous system, complex and heterogeneous from the clinical point of view. The aetiology of MS is controversial and the underlying molecular mechanisms are still not completely understood. Gait dysfunction is one of the most debilitating symptoms in MS. Approximately 85\% of patients indicate impaired gait as one of the major limitations in their daily life.

Acupuncture, as a therapeutic form of Traditional Chinese Medicine (TCM), regulates the vegetative functions to maintain homeostasis by stimulating self-regulation mechanisms. In some acupuncture studies a reduction of spasticity and improvement of fatigue and imbalance has been found in MS patients, but there is a lack of studies regarding gait.

Material and Methods: Based on the Heidelberg model of TCM, an acupuncture prospective, randomized and controlled study in cross-over design was done in order to improve gait problems in patients with MS. The sample consisted of 20 individuals, all of them with a diagnosis of MS of relapse-remitting type (RRMS). Gait impairment was evaluated by the T25FW (25-Foot test), a timed walking over a fixed distance measure, recommended for clinical trials in MS. Patients were blinded and randomized into one of two groups (A and B). The acupuncture intervention was divided into two moments: in the first one, group A of patients received a true acupuncture treatment, using ST34, Bl40 and Bl57 points, and patients of group B received sham acupuncture treatment. At a second time, group A received sham acupuncture and group $B$ true acupuncture. Before and after each treatment, gait impairment evaluation was done.

Results: The results of the T25FW test, comparing before and after acupuncture, showed statistically significant differences $(p<0.05)$ in true acupuncture, when 
compared with sham acupuncture. We also found that when using true acupuncture, $95 \%$ of cases show an improvement in $\mathrm{T} 25 \mathrm{FW}$ test, compared to $45 \%$ when sham acupuncture was done.

Conclusions: The results obtained in the present study can support the idea that the T25FW test is sufficiently sensitive to assess the effect of treatment and so can be a useful tool as a clinical measure of gait in studies involving acupuncture. Our study protocol provides evidence that acupuncture treatment, according to Heidelberg model of TCM, can result in improvements in mobility. This makes acupuncture an attractive option for intervention for MS patients with gait impairment looking for effective therapy.

Keywords: Gait, Multiple sclerosis, T25FW test.

\section{Immediate Effect of Acupuncture on Pain and Range of Motion in Patients with Shoulder Pain}

\begin{abstract}
Mario Costa1, Gonçalo Borges ${ }^{2}$, Nuno Correia ${ }^{3}$, Maria João Santos ${ }^{1}$, Bruno Ramos ${ }^{1}$, Jorge Machado ${ }^{1,5}$, Henry Johannes Greten ${ }^{1,4}$

${ }^{1}$ TCM Master Programme Abel Salazar Institute for Biomedical Sciences (ICBAS), University of Porto, Porto, Portugal; ${ }^{2}$ Physical and Rehabilitation Medicine Service, Hospital of Prelada, Santa Casa da Misericórdia, Porto, Portugal; ${ }^{3}$ Acupuncture Clinic, Gaia Private Hospital, Porto, Portugal; ${ }^{4}$ Heidelberg School of Chinese Medicine, Heidelberg, Germany; ${ }^{5}$ Labiomep-Porto Biomechanics Laboratory - University of Porto, Porto, Portugal
\end{abstract}

Background: Shoulder pain (SP) is a frequent condition that leads to functional impairment as well as high individual and social costs. There is a worldwide prevalence of 7 to $25 \%$ of SP which represents $12 \%$ of chronic pain in Portugal. Acupuncture is widely used against chronic pain with low cost and little side effects. We were interested in a comparison of two acupuncture regimens in order to optimize treatment.

Objectives: The purpose of this study is to assess the immediate effect of acupuncture in the shoulder pain and range of motion.

Methodology: 32 patients were divided in 2 groups by random. Subjective perception of pain was assessed by VAS and objective measurement of the amplitude of abduction was carried out before and 5 minutes after acupuncture to assess its clinical effects. Group A received acupuncture on C3 and It11 and group B on F21 and an extra point located 5 cun above elbow. Pain reduction and ROM was assessed and statistically compared by Student's t test.

The study included male or female patients, aged between 18-85 years old, with pain in shoulder and limitation of shoulder abduction movement, diagnosed by an independent physiatrist with no previous experience of acupuncture and the TCM diagnosis of a yang maior Syndrome. Exclusion criteria involved patients with shoulder pathologies without pain in abduction, patients with neurological or oncological pathologies, pregnant and infants.

Identical depth and intensity of stimulation of acupuncture were applied in both groups using Leopard-spot technique.

Results: (1) Before acupuncture both groups showed similar demographic and clinical characteristics; (2) In group A pain improved $39.01 \%$, resulting in a statistically significant pain reduction $(\mathrm{p}<0.001)$. The amplitude improved by an average of 28.57 degrees, showing statistically significant data; (3) The comparison of group A and B revealed that pain reduction was significant in group $A$ and has increased in group B. The ROM improvement in EG was twice the one of the CG.

Conclusion: Acupuncture according to the Heidelberg (HD) Model resulted in significantly immediate pain reduction. Also the ROM doubled in the HD model rather than in the control group. Acupuncture is a technique with immediate effect in painful shoulder.

Keywords: Shoulder pain, range of motion.

\section{Comparison of Two Acupunctures Strategies on Functional Dyspepsia}

Susana Martins ${ }^{1}$, Maria João Santos ${ }^{1}$, Jorge Machado ${ }^{1,2}$,
Henry Johannes Greten ${ }^{1,3}$
${ }^{1}$ TCM Master Programme, Abel Salazar Institute
for Biomedical Sciences, University of Porto, Porto,
Portugal; ${ }^{2}$ Labiomep-Porto Biomechanics Laboratory -
University of Porto, Portugal; ${ }^{3}$ Heidelberg School of
Chinese Medicine, Heidelberg, Germany

Introduction: Dyspepsia is a benign chronic disorder, and can be defined as a pain or discomfort located in the upper abdomen, excluded reflux symptoms.

Functional Dyspepsia, according to the Rome III criteria, is a symptom complex characterized by epigastric pain or burning, postprandial fullness or early satiety, bloating in the upper abdomen, nausea, vomiting, belching and absence of organic diseases in upper endoscopy. About twenty percent of adults in the western world have functional dyspepsia.

Objectives: The purpose of this study is to use acupuncture as an additive to regular pharmacology treatments in patients with Functional Dyspepsia. The objective is compare the clinical effect between acupuncture at special acupoints and non-specific acupoints on functional dyspepsia.

Methodology: Prospective randomized control trial in parallel group design $(n=2 \times 30)$. A group will be treated with treatment A - acupuncture at special acupoints (leopard spot technique) - Verum Acupuncture, and a second group with treatment B - acupuncture at non-specific acupoints. 
Intervention: (1) Group A - Treatment A - bloodletting Rs13, H2, S44, (2) Group B - Treatment B - acupuncture at non-specific acupoints.

Parameters: (1) Score dyspepsia symptoms, (2) Evaluation of myoelectric activity of the stomach through the electrogastrogram ( $3<$ clinical score $>3$ ), (3) Algometer (maximum tolerable permission on G2). Values recorded will be compared before and after treatment.

Expected Results: Results prove the efficacy of acupuncture in the treatment of Functional Dyspepsia and is superior to non-specific acupoints.

Keywords: Functional dyspepsia - algometry - electorgastrogram.

\section{The Impact of Acupuncture on Improving Gait in Patients with Multiple Sclerosis}

Ricardo Loureiro ${ }^{1}$, Maria João Santos ${ }^{1}$, Nuno Correia ${ }^{1,2}$, Jorge Machado ${ }^{1,3}$, Henry Johannes Greten ${ }^{1,4}$

${ }^{1}$ TCM Master Programme, Abel Salazar Institute for Biomedical Sciences, University of Porto, Porto, Portugal; ${ }^{2}$ Acupncture Clinic, Alfena Private Hospital, Porto, Portugal; ${ }^{3}$ Labiomep-Porto Biomechanics Laboratory - University of Porto, Portugal; ${ }^{4}$ Heidelberg School of Chinese Medicine, Heidelberg, Germany

Background: Multiple Sclerosis is a complex autoimmune disease mediated by an immune response to central nervous system antigens. It is a neurodegenerative disease and often progresses to loss of physical and cognitive abilities, including disorders of the gear.

Acupuncture is one of the therapeutic techniques of Traditional Chinese Medicine that helps to improve the relief of symptoms resulting from Multiple Sclerosis, particularly in terms of gear.

Objectives:

Overall Objective:

- Promote improved quality of life for patients with Multiple Sclerosis.

Specific Objectives:

- Identify the benefits of using acupuncture in patients with Multiple Sclerosis;

- Identify the influence of the use of acupuncture in the gait of patients with Multiple Sclerosis;

- Encourage the use of acupuncture in patients with Multiple Sclerosis.

Methodology: For the study, patients were enrolled in selected National Multiple Sclerosis Association with the following inclusion criteria: diagnosis of Multiple Sclerosis for at least five years; patients who do not use any orthosis for gait and without cognitive impairment. The patient was registered running time on a straight path 30 meters. The duration of the gear was made before the therapeutic with acupuncture and after 16 sessions.
Results: After 16 sessions of acupuncture, the duration of the gear was lower than before the first session.

Conclusion: This study concludes acupuncture efficiency improve walking in patients with Multiple Sclerosis. So, we suggest use of acupuncture in the treatment of patients with Multiple Sclerosis, at hospitals, medical centers and patients associations.

Keywords: Multiple sclerosis, gait, QoL.

\section{Acupuncture Injection Point Therapy for Urinary Incontinence in Adults after Stroke: Prospective Randomized Blinded Placebo Controlled Trial - A Research Protocol}

Rui Pedro Loureiro ${ }^{1}$, Maria João Santos', Jorge Machado ${ }^{1,2}$, Henry Johannes Greten ${ }^{1,3}$

${ }^{1}$ TCM Master Programme, Abel Salazar Institute for Biomedical Sciences, University of Porto, Porto, Portugal; ' Labiomep-Porto Biomechanics Laboratory University of Porto, Portugal; ${ }^{3}$ Heidelberg School of Chinese Medicine, Heidelberg, Germany

Background: After Stroke, a large percentage (40-60\%) of the people admitted to hospital develop urinary incontinence, even on hospital discharge still $25 \%$ of that patients suffers from urinary incontinence and $15 \%$ still remain incontinent at one year.

Objectives: To verify the efficacy of the acupuncture injection point therapy for urinary incontinence in adults after stroke.

Methodology: This will be a prospective study designed to assess the action of the acupuncture injection point therapy for urinary incontinence in adults after stroke in three randomly assigned groups of patients. Will be observed $(\mathrm{n}=$ 30 , each). The verum group will be treated with acupuncture injection therapy in acupuncture point cella secunda (vesical conduit 32 - Ci Liao) using a saline solution plus vitamin B1 and the placebo group using acupuncture injection therapy in the same point only with saline solution, and the third group will be in the control group.

The treatment impact will be assessed by Incontinence Impact Questionnaire (IIQ 7) and Urogenital Distress Inventory (UDI 6) before and after each course of treatment.

Inclusion Criteria: Male or Female adults who suffer stroke and develop urinary incontinence after stroke and those who signed the informed consent.

Intervention: 5 days of treatment will be a course treatment; patients will do maximum 3 courses.

Expected Results: It is hoped that treatment with Acupuncture injection therapy, improve the patient's quality of life, decreasing the episodes of urinary incontinence.

Keywords: Urinary incontinence, stroke, acupuncture injection therapy. 


\section{Acupuncture Efficacy for Shoulder Pain in Adults after Stroke: Prospective Randomized Blinded Placebo Controlled Trial - A Study Protocol}

Rui Pedro Loureiro ${ }^{1}$, Maria João Santos ${ }^{1}$, Jorge Machado ${ }^{1,2}$, Henry Johannes Greten ${ }^{1,3}$

${ }^{1}$ TCM Master Programme, Abel Salazar Institute for Biomedical Sciences, University of Porto, Porto, Portugal; ${ }^{2}$ Labiomep-Porto Biomechanics Laboratory University of Porto, Portugal; ${ }^{3}$ Heidelberg School of Chinese Medicine, Heidelberg, Germany

Background Information: After stroke, hemiplegic shoulder pain affects from $16 \%$ to $72 \%$ of the patients. This condition causes a great amount of distress and reduced activity.

Objectives: To verify the efficacy of the acupuncture point Stomach 38 (Tiao Kou) plus extra conduit point zhongping and scalp acupuncture using Heidelberg model in adults with hemiplegic shoulder pain after stroke.

Methodology: This will be a prospective study designed to assess the action of the acupuncture point Stomach 38 (Tiao Kou) and extra counduit point zhongping in adults with hemiplegic shoulder pain after stroke in four randomly assigned groups of patients.

Will be observed ( $n=30$, each). The verum group 1 will be treated with acupuncture point Stomach 38 (Tiao Kou) and extra conduit point zhongping, the verum group 2 will be treated with scalp aucpunture using the Heidelberg model, the placebo group using sham acupuncture, and the third group will be in the control group.

The treatment impact will be assessed by Disability of the Arm, Shoulder, and Hand scale (DASH), before and after each course of treatment.

Inclusion Criteria: Male or Female adults who suffer stroke and develop hemiplegic shoulder pain after stroke and those who signed the informed consent.

Intervention: 5 days of treatment will be a course treatment; patients will do maximum 3 courses.

Expected Results: It is hoped that treatment with acupuncture, improves the patient's quality of life, decreasing the shoulder pain and improving the mobility.

Keyword: Shoulder pain, stroke, QoL.

\section{Immediate Effect of Acupuncture on Post-Operative Pain after Lumbar Surgery}

Fatine Hamza1, Ana Anjos', Jorge Machado 1,2, Manuel Laranjeira ${ }^{1}$, Henry Johannes Greten ${ }^{1,3}$

${ }^{1}$ TCM Master Programme, Abel Salazar Institute for Biomedical Sciences, University of Porto, Porto, Portugal; ${ }^{2}$ Labiomep-Porto Biomechanics Laboratory University of Porto, Portugal; ${ }^{3}$ Heidelberg School of Chinese Medicine, Heidelberg, Germany

Background: Acupuncture has a wide range of possible applications and may be able to contribute to the management of post-operative pain. Moreover, in general, side effects from acupuncture are low. It therefore represents a useful therapeutic option to consider within the overall pain management of individuals.

Aim: To assess the effect of acupuncture on pain after lumbar surgery.

Methods: We designed a randomized single-blinded controlled clinical trial to investigate if acupuncture can be a useful therapeutic option in post-operative pain after lumbar surgery.

30 patients with post-operative pain after lumbar surgey were randomly assigned to two groups. Group one: Verum group received acupuncture in three points R10(KI10), V40(BL40) and L6(SP6). Group two: received Sham acupuncture in three points that does not belong to any meridian. Leopard spottechnique was the chosen acupuncture technique for both groups. Pain was evaluated by subjective measurement using the VAS and objectively by measuring the angle of the hip in the straight leg raise test (Modified Lasegue Test) to the painful point of the active movement, before and after the acupuncture.

Results: Our results showed a significant decrease in the pain on VAS by almost half comparing to before the acupuncture in both groups. A significant increase in angle measurement was noted as well between before and after acupuncture in both groups. By comparing data in both groups we found out that the verum group showed better results than the sham group. However, this difference was not statistically significant at $\mathrm{p}>0.005$.

Our data suggests that acupuncture can be used as a postoperative pain analgesic. It also proved the efficiency of the points choice. However, the therapeutic effect found in the control group is not unusual. Sham acupuncture appeared to have an analgesic effect in $40 \%$ to $50 \%$ of patients compared to $60 \%$ to $75 \%$ for real acupuncture.

Conclusion: Acupuncture showed to be an effective way of treatment in post-operative pain after lumbar surgery for LDD and LSS. No side effects for acupuncture were found in our study. A significant difference was found before and after the acupuncture in patients who were consuming regularly their conventional analgesic medication. The subjective and objective methods of measurements were both improved after acupuncture. Finally, acupuncture can be used effec- 
tively as an adjunct to conventional analgesia in surgery departments.

Keywords: Postoperative pain, lumbar surgery, straight leg test, Lasegue test.

\section{Qigong at School as a Method to Control Teachers' Anxiety, Stress and Depression}

Renato Oliveira ${ }^{1}$, Mário Gonçalves ${ }^{1,2}$, Jorge Machado ${ }^{1,3}$, Henry Johannes Greten ${ }^{1,2}$

${ }^{1}$ TCM Master Programme, Abel Salazar Institute for Biomedical Sciences, University of Porto, Porto, Portugal; ${ }^{2}$ Heidelberg School of Chinese Medicine, Heidelberg, Germany; ${ }^{3}$ Labiomep-Porto Biomechanics Laboratory - University of Porto, Portugal

Background: Anxiety has become a big problem in the general population environment and consequentially it became an issue at school as well. If the practice of qigong, a traditional vegetative feedback exercise system, could reduce anxiety and its multiple consequences, this could potentially improve the wellbeing of teachers as well as reduce the cost of respective health care society.

Objectives:

General Objectives: (1) To evaluate the potential effect of Qigong exercises in teachers' anxiety, stress and depression; (2) To evaluate the potential influence of the practice of Qigong and the functioning of the Autonomic Nervous System (ANS).

Specific Objectives: (1) To check teachers' anxiety, stress and depression levels; (2) To evaluate the effectiveness of the 'White Ball Qigong' exercises in changing teachers' levels of anxiety, stress and depression; (3) To evaluate the influence of Qigong in ANS (measured by HRV) in school context.

Methodology: The aim of this prospective, randomised, placebo-controlled, blinded clinical study was to test the effect of a specific set of physical exercises of Qigong ('White Ball') in two groups of teachers: a verum and placebo group (placebo Qigong).

Intervention: The verum group got real Qigong classes, the sequence of exercises known as 'White Ball Qigong', while the control group did placebo qigong. Measures were done using psychometric and physiological parameters.

Results: After the intervention, the Verum group presented significant Anxiety Trait results (A-T) $[p=0.004]$; A-S $(p<0.01)$; EADS-Anxiety (EADS-A) [p = 0.002]; EADSDepression (EADS-D) [p = 0.001]; EADS-Stress (EADS-S) $[\mathrm{p}<$ 0.001]; Analogical Visual Scale for anxiety (AVS) [ $p=0.013]$; SDNN ( $\mathrm{p}=0.003$ ); sympathetic index (IS) [p < 0.001]; parasympathetic index (IR) $[\mathrm{p}<0.001]$ and Heart Rate (HR) $[\mathrm{p}=$ $0.002]$. The control group presented significant results in IS ( $p=0.017)$ and IR ( $p=0.017)$, although in this case this corresponds to negative scores. A comparison of both groups shows significant differences in the A-S $(p=0.005)$ and IS $(p=0.045)$ variables.
Conclusion: 1) Qigong had positive effects in the ANS; 2) anxiety is a problem among teachers; 3 ) the benefit from Qigong exercises is a reduction in A-S and A-T and stabilization of the vegetative functions; 4) A-S was increased by stressors continuous action, through a longitudinal comparison; 5) a parallel group comparison reveals that the trait of anxiety was different yet not significantly, possibly due to short therapy or the sample size.

Assuming that values up to 35 (STAI scale) are considered normal, we assume that from that value on, each point increase indicates that anxiety became pathological. Therefore, we underline the importance of anxiety reduction; pathological A-T was reduced in average and relative terms in approximately $2 / 3(14 \%)$ and A-S was totally reduced $(30 \%)$, which may indicate a clinically relevant reduction of anxiety.

Keywords: Qigong, anxiety, teachers, Chinese Traditional Medicine and Heart Rate Variability.

\section{A Study of the Efficacy of Radix Berberis Vulgaris on Acute States of Atopic Dermatitis}

\author{
Paula Pinho ${ }^{1}$, Maria João Santos ${ }^{1}$, Jorge Machado ${ }^{1,2}$, \\ Henry Johannes Greten ${ }^{1,3}$ \\ ${ }^{1}$ TCM Master Programme, Abel Salazar Institute \\ for Biomedical Sciences, University of Porto, Porto, \\ Portugal; ' 2 Labiomep-Porto Biomechanics Laboratory - \\ University of Porto, Portugal; ${ }^{3}$ Heidelberg School of \\ Chinese Medicine, Heidelberg, Germany
}

Background/Introduction: Atopic Dermatitis Syndrome (ADS) is a chronic cutaneous inflammatory disease whose pathogenic mechanism is unknown. There are few epidemic studies of the incidence of ADS in Portuguese population. The common (Western Medicine) therapeutic formulations used in ADS, besides of emollient formulations, can be aggressive to the patient, because of the lateral effects.

Objectives: The purpose of this study is to improve the clinical state of patients and to harmonize the therapeutic effects of Conventional and Chinese medicines on Atopic Dermatitis Syndrome, and also to assess the clinical efficacy of the Chinese herbal topic formula for treating this disease.

Methodology: Interventional, randomized, prospective and controlled study.

Intervention: The patient sample was divided in Group A which was treated with an ointment of Radix Berberis and Group B which received an ointment without Radix Berberis.

Main Parameter: Gravity of the eczema for the last 3 days, by PO SCORAD* (Patient Oriented Scorad) for disease activity assessment as a simple, validated, reproducible, non age-dependent test.

Secondary Parameter: Diameter of the treated skin lesion.

Expected Results: After treatment, it is expected that all parameters recorded in Group A will be equal or better than 
those in Group B. It is also expected that this study can be used to improve the patient's quality of life and to reduce the damage by drugs, such as secondary effects in the human body. By doing so, the Portuguese Public Health Service (Serviço Nacional de Saúde) could reduce the costs of hospital admissions and medical appointments.

Keywords: Radix Berberis, atopic dermatitis.

\section{Comparison of Two Methodologies on Weight Loss in Traditional Chinese Medicine Therapy}

\author{
Diogo Matos ${ }^{1}$, Andreia Vieira ${ }^{1}$, Maria Jõao Santos ${ }^{1}$, \\ Jorge Machado ${ }^{1,2}$, Jürgen Stürmer ${ }^{1,3}$, \\ Henry Johannes Greten ${ }^{1,3}$ \\ ${ }^{1}$ TCM Master Programme, Abel Salazar Institute \\ for Biomedical Sciences, University of Porto, Porto, \\ Portugal; ' 2 Labiomep-Porto Biomechanics Laboratory - \\ University of Porto, Portugal; ${ }^{3}$ Heidelberg School of \\ Chinese Medicine, Heidelberg, Germany
}

Abstract: Nowadays the implications of obesity, predominantly abdominal fat, such as hypertension, diabetes, dyslipidemia, and cardiovascular disease are well known in the general population. It would be desirable to control obesity by safe and effective treatment modalities, and among the different methods acupuncture is one of the most popular complementary treatments.

Objective: A randomized controlled clinical trial to examine the effectiveness of auricular acupuncture on body weight loss in a Portuguese population.

Methodology: After a pre-study, an experimental crosssectional, prospective, randomized, controlled and singleblinded study will be conducted. This sample includes adults $(\mathrm{n}=60$ ) from 3 different gymnasiums between 25 and 55 years of age. In the population of the study a questionnaire was used to record the physical activity, and an informed consent had been obtained. In addition, a low carb diet and auricular acupuncture was used. The study population was divided in two verum and one control groups. All three groups follow a prescribed diet by a nutritionist.

Group 1: Standardized acupoints were selected on the external ear according to Chinese Medicine and clinical experience: Shenmen (TF4), stomach (CO4), hunger point, mouth (C01), center of ear (HX1), and Sanjiao (C017). Acupuncture in the treatment group was applied with routine ear pressing plasters (Vaccaria ear seeds, Beijing Zhongyan Taihe Medicine, Beijing, China).

Group 2: Heidelberg methodology: Standardized acupoints were selected on the external ear according to Chinese Medicine and clinical experience, using ASP gold needles, Strengthening the center by Point zero (the navel) dominant ear, Porta fortunae non-dominant ear, Barbiturate point non-dominant ear, Psychosomatic key point dominant ear and the point against aggression.
Group 3: control group, only followed a prescribed diet therapy.

Main and Second Parameters: Weight/body composition by bioelectrical impedance analysis, hight and abdominal/hip perimeter, weight and IMC.

Keywords: Waist-to-Height ratio, Body Mass Index, Abdominal obesity, acupuncture.

\section{Improvement of Menopausal Symptoms Using a TCM Approach - The Effect of Individualized Acupuncture and Phytotherapy as Assessed by the Menopause Rating Scale}

Nuno Correia ${ }^{1,2}$, Vânia Alves Barros ${ }^{1,2}$, Maria João Santos ${ }^{2}$, Henry Johannes Greten ${ }^{2,3}$

${ }^{1}$ Acupuncture Clinic, Alfena Private Hospital, Porto, Portugal; ${ }^{2}$ TCM Master Programme, Abel Salazar Institute for Biomedical Sciences, University of Porto, Porto, Portugal; ${ }^{3}$ Heidelberg School of Chinese Medicine, Germany

Background: Long term use of hormone replacement therapy involves a health risk, and many women seek alternative strategies to relieve climacteric complaints. Previous studies suggest that acupuncture may alleviate menopauserelated symptoms, such as hot flashes. On the other hand, Er-Xian decoction, a formula of Chinese medicine, has been shown to be effective in treating menopausal symptoms, but more studies with improved designed are needed.

Case Description: A 50 year-old female patient, in perimenopause for one year, was referred by her gynaecologist for acupuncture to treat her severe menopause symptoms, after no improvement with phytoestrogens or anxiolytic drugs. The patient refused any other conventional treatments, including hormonal replacement therapy. Her main symptoms were: severe hot flushes, profuse sweating at night, palpitations, chest oppression, intermittent hyperthermia, insomnia, joint complaints and emotional lability. Hyperthyroidism was excluded.

After a TCM diagnosis based on the Heidelberg Model of TCM, the patient was initially submitted to weekly body acupuncture (main points: SP6, SP10; LI14; HT7; GB21; GB 20; TE17; ST36; L3) as well as ear acupuncture (uterus and shen men points) during two months and started on Er-xian herbal formula ('Two Immortals formula'). Clinical response was monitored using the Menopause Rating Scale (MRS), developed by the Berlin Center for Epidemiology and Health Research), a 11-item questionnaire with a maximum score of 44 , filled in by the patient herself with no interference from the practitioner. After 7 sessions of acupuncture, the MRS lowered from a baseline of $31 / 44$ to $12 / 44$ (61\% reduction), and the patient was then submitted to 2 -weekly acupuncture treatments. At the end of 17 sessions ( 8 months), a minimum 
MRS was achieved (MRS $=4 / 44$ ), showing a clinical improvement of $87 \%$. The patient remains on monthly followup treatments and a sustained reduction of symptoms is observed (MRS of 3 to 4), showing clinical stability. No side effects were seen.

Conclusion: Acupuncture and Chinese Phytotherapy may represent an alternative to conventional treatments, with no side effects. We believe that an individual TCM diagnosis is needed for a successful clinical outcome. This case report showed a marked reduction of symptoms (61\% at 2 months and $87 \%$ at 8 months) on a severely affected woman. We recommend the use of measurement tools, such as the MRS, in order to monitor clinical responses and lessen practitioner bias on the clinical approach.

Keywords: Menopause Rating Scale, menopausal symptoms, Two immortals formula.

\section{Acupuncture May Be Beneficial on Delayed Onset Muscle Soreness: Results from a Preliminary Study}

\author{
Ricardo Cardoso ${ }^{1}$, Nuno Correia ${ }^{1,2}$, Maria João Santos ${ }^{1}$, \\ Henry Greten ${ }^{1,3}$ \\ ${ }^{1}$ Master in Traditional Chinese Medicine, Abel Salazar \\ Institute for Biomedical Sciences, University of Porto, \\ Porto, Portugal; ${ }^{2}$ Acupuncture Clinic, Private Hospital of \\ Gaia, VN Gaia, Portugal; ${ }^{3}$ Heidelberg School of Chinese \\ Medicine, Germany
}

Introduction: Despite of the many studies on the subject of delayed onset muscle soreness (DOMS), the explanation of the mechanisms underlying this clinical condition is still under discussion, as well as the strategies for its prevention and treatment. Scientific studies have shown no consensus of the effects of acupuncture on DOMS.

Objective: To evaluate acupuncture effects on DOMS.

Methods: 45 participants (mean $\pm \mathrm{s}$ age $25.38 \pm 4.77$ years; weight $65.76 \pm 9.88 \mathrm{~kg}$; height $169 \pm 0.09 \mathrm{~cm}$ and body mass index $22.94 \pm 2.51 \mathrm{~kg} / \mathrm{m}^{2}$ ) of both genders (19 males, 26 females), after completing a screening questionnaire and providing written informed consent, were randomly distributed into three groups (verum acupuncture group, sham acupuncture group and a control group - VA, SA and CG). Muscle soreness (MS), pressure pain threshold (PPT), range of motion (ROM), vertical jump (VJ) and Isokinetic evaluations were performed before and after (immediately and $24 \mathrm{~h}$ ) an exercise induced muscle damage (EIMD) protocol which subjects performing a five sets of 20 drop jumps from a height of $0.6 \mathrm{~m}$ step, with a 10 seconds interval between jumps and 2 minutes rest period between sets. Statistical analysis was performed using Microsoft Excel 2010 to determine the mean and standard deviations of the sample and outcome measures, and IBM SPSS Statistics 22 was used to perform a KruskalWallis test with for analysis of differences between groups in the different moments and a Friedman test with a Bonferroni post-hoc test, to assess differences within the group along the different moments.

Results: The protocol of EIMD showed success in inducing DOMS. When VA, SA and CG groups were compared, it was found that VA had better results in all outcome measures, especially in MS, PPT and VJ. However, SA group showed better results than CG, when MS and PPT were compared, but they were not statistically significant.

Conclusion: The results showed that acupuncture applied after an EIMD protocol, had an effect on MS reduction and an improvement on PPT, ROM, VJ and Isokinetic measures.

\section{Overcoming the Double-Blinding Problem in Acupuncture Research: A Novel Methodology Successfully Applied on a Lower Back Pain Clinical Study}

\author{
Alcino Duarte ${ }^{1}$, Nuno Correia ${ }^{2,7,9}$, Márcia Sá ${ }^{3}$, \\ Jorge Machado ${ }^{4,6}$, Eusébio Laranjeira ${ }^{5}$, \\ Manuel Laranjeira ${ }^{6}$, Henry Johannes Greten ${ }^{6,8}$ \\ ${ }^{1}$ Master in Traditional Chinese Medicine, Abel Salazar \\ Institute for Biomedical Sciences, University of Porto, \\ Porto, Portugal; ${ }^{2}$ Nursing School of Porto, University of \\ Porto, Porto, Portugal; ${ }^{3}$ Health in Family' Health Care \\ Unit, Porto, Portugal; ${ }^{4}$ Porto Biomechanics Laboratory \\ (LABIOMEP), University of Porto Abel Salazar Institute \\ for Biomedical Sciences, Porto, Portugal; ${ }^{5}$ Institute for \\ Neurosciences, Porto, Portugal; ${ }^{6}$ Tradicional Chinese \\ Medicine Master Programme, Abel Salazar Institute \\ for Biomedical Sciences, University of Porto, Porto, \\ Portugal; ${ }^{7}$ General Pathology, Nursing School of \\ Porto, University of Porto, Porto, Portugal; ${ }^{8}$ Heidelberg \\ School of Chinese Medicine, Heidelberg, Germany; \\ ${ }^{9}$ Acupuncture Clinic, Alfena Private Hospital, Porto, \\ Portugal
}

Introduction: Double-blinding remains a limitation on acupuncture clinical research. Pre-existing studies on lower back pain (LBP) reveal the contradictory mosaic of findings on whether acupuncture may be useful in this condition. In an attempt to overcome these limitations, a double-blinding assay was developed for a clinical study on acupuncture for LBP.

Materials and Methods: Sixty-seven patients with LBP and 'Yang Major Syndrome' were enrolled in a randomized, controlled clinical trial with a novel double-blinded study design. Individuals were allocated into a verum group (VG, true acupoints, $n=33$ ) and a control group (CG, non-acupoints, $\mathrm{n}=34$ ). Outcomes were back flexion measured by inclinometry and pain assessed by visual analogue scale.

Results: Flexion improved in the VG (mean $17.6 \pm 9.7^{\circ}$, $\mathrm{p}<0.0001)$ and not in the CG $\left(3.8 \pm 8.6^{\circ}, \mathrm{p}<0.0001\right)$ (fig. 1). Pain score reduced in the VG (mean $36.6 \pm 17.9 \%, p<0.0001$ ) while in CG no difference was found $(12.6 \pm 15.2 \%, p=0.890)$ 


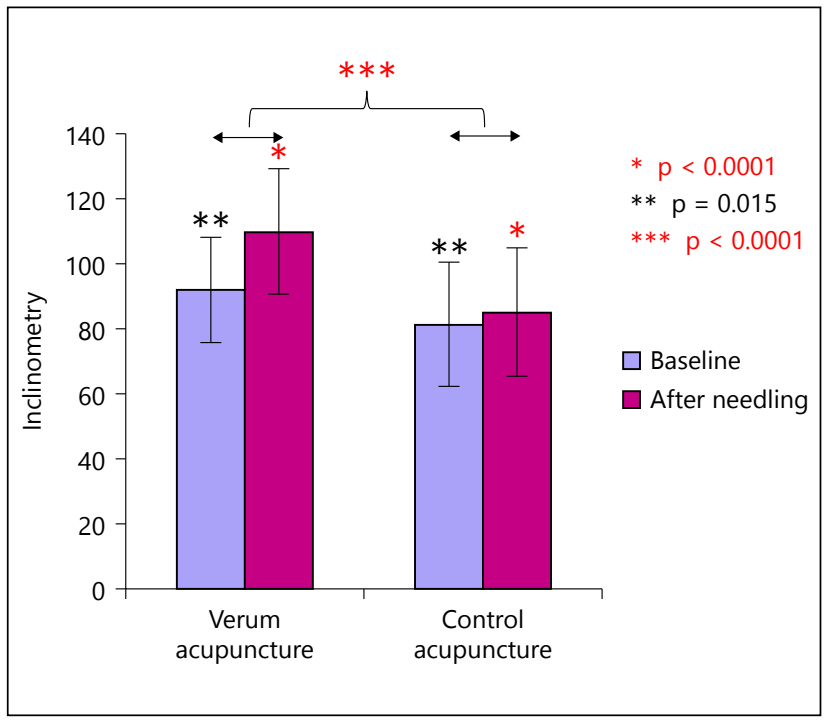

Fig. 1. Objective assessment: verum group: 17.6 degrees more flexion (vs. control 3.8 degrees). Increase of mobility of $19.7 \%$ in the verum group vs. $5.5 \%$ in the control group.

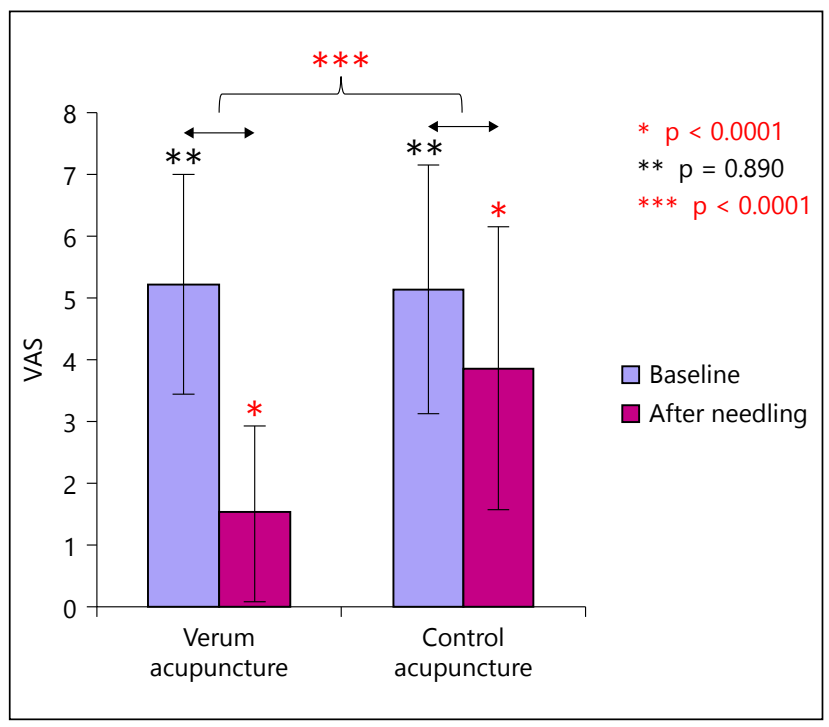

Fig. 2. Subjective Pain Reduced Significantly in the Verum Group (36.6\%) vs. Control Group (12.6\%).

(fig. 2). These findings equaled a mean increase on mobility of $19.7 \pm 11.1 \%$ in the VG vs. $5.5 \pm 11.9 \%$ in the CG ( $p<0.0001)$.

Conclusion: This is a novel double blinding design in acupunctures studies for LBP. It shows that acupuncture based on a TCM diagnosis and assessed by objective parameters has significant and specific effects. Results support the need for long-term evaluation of acupuncture in LBP based on this blinding assay.

\section{Qigong Improves Attention Level on School Kids: Results from a Novel Placebo-Controlled Qigong}

\author{
Leonel Duarte ${ }^{1}$, Mário Gonçalves 1,2,3, Jorge Machado1,5, \\ Patrícia Mendes ${ }^{4}$, Petra Froeschen ${ }^{2,3}$, Henry J. Greten ${ }^{1,2,3}$ \\ ${ }^{1}$ ICBAS - Abel Salazar Institute for Biomedical \\ Sciences, University of Porto, 4099-030 Porto, \\ Portugal; ${ }^{2}$ DGTCM - German Society of \\ Traditional Chinese Medicine, 69126 Heidelberg, \\ Germany; ${ }^{3} \mathrm{HSCM}$ - Heidelberg School of Chinese \\ Medicine, 69126 Heidelberg, Germany; ${ }^{4}$ UM-EP \\ University of Minho - School of Psychology 4710 \\ 057 Braga, Portugal; ${ }^{5}$ LABIOMEP - Biomechanics \\ Laboratory of Porto, University of Porto, 4200-450 \\ Porto, Portugal
}

Background: Qigong is an exercise system of Traditional Chinese Medicine that may be regarded as a neurovegetative biofeedback therapy. The ability to direct and maintain attention is a prerequisite for learning, and a lack of attention may affect school performance, a problem for which a solution is far from being found. Qigong exercises are already applied to increase attention and memory capacity. However, there is a lack of scientific data showing an effect of Qigong on attention.

Objectives: 1) To evaluate the effects of White Ball Qigong on attention levels of adolescents (12-14 years) at school and to demonstrate the feasibility of such training in the context of everyday's school life.

Methods: A prospective, controlled, randomized and single blinded clinical study was carried out in a parallel group design. For this purpose a special control group (placebo Qigong) was developed to serve as a control. $N=66$ adolescents of three classes attending the eighth highschool grade were recruited (one complet highschool year). The three classes were randomly allocated to a verum group $(n=22)$, control group (placebo Qigong) $(n=22)$ and waiting list group $(n=22)$ after written consent according to the Helsinki declaration. No participant ever had any previous experience in Qigong.

Intervention: Verum and control group (placebo Qigong) received training in the exercises with a duration of 5 minutes at the end of the Physical Education lessons 2x/week. Both groups were encouraged to repeat the exercises daily at home. Interviews twice a week revealed that the exercise frequency was statistically equal in both groups.

Control group (placebo Qigong) consisted of a standing position while watching a television program of their choice. Students were informed that this activity was a valid way to improve their attention. The waiting list group was left without any intervention.

Attention Tests $\left(D_{2}\right)$ were applied before the exercises ( $\mathrm{t} 1$, pre-test), and after two and four weeks ( $\mathrm{t} 2$, post-test and t3, follow-up). This test measures: Total Number of Items Processed (TN); Total Hits (TH); Total Number of Items Minus 
Error Scores (TN-E); Concentration Performance (CP) and Fluctuation Rate (FR).

Results: At t1, there was no significant difference between the 3 groups in all 5 measurable features. At $t 3$, the verum group was significantly superior in all 5 measurable features in comparison to the control group (placebo Qigong) and waiting list group. $p$ values differed for TN ( $p=$ $0.000)$, TH $(p=0.000)$ TN-E $(p=0.023), \mathrm{CP}(p=0.004)$, and FR $(p=0.003)$. Values in the waiting list group were generally worse than in the control group (placebo Qigong), but there was no significant difference between control group (placebo Qigong) and waiting list group, indicating a relatively low effect in comparison of the group with no intervention.

Conclusion: One of the main problems of Qigong studies is the buildup of adequate methods of control. To the best of our knowledge, this study was the first placebo-controlled Qigong study to evaluate Qigong effects. Qigong may be recommended to improve attention levels of the students, an option with low cost and no side effects, as opposed to current conventional treatments.

\section{Integration of East-West Approaches to Emotionality - A Step Towards the Parametrization of the TCM Concept of 'Constitution'}

\author{
Carla Alexandra Paiva ${ }^{1,2}$, Henry Johannes Greten ${ }^{1}$ \\ ${ }^{1}$ University of Minho, Braga, Portugal; ${ }^{2}$ University of \\ Porto, Instituto de Ciências Biomédicas Abel Salazar, \\ Porto, Portugal
}

Introduction: Emotion is conceptualized as a self-regulatory homeostatic mechanism with impact on brain and organ systems functioning, and consequently involved in disease ethiopathogenics. The pioneer work of William James theory on 'cortical readout' feedback of emotion specificity and the recent neuroscience findings supporting its empirical validation permits a translational research of East and West medicine concepts. The current understanding of TCM emphasizes the original logical access inherent in Chinese Medicines to body-mind homoeostasis, however the translation to western concepts is need to its better application in healthcare practices.

Methods: A systematic review on major postulates on two east-western emotion theories considering neurobiological and psychophysiological correlates. To compare 'modern cortical readout theories' on emotion with a TCM based model - the Heidelberg Model, regarding the etiopathogenic role of emotion on psychosomatic diseases.

Results: The models are susceptible to be comparable in their major assumptions. In fact, neuroscience findings show that emotion and cognition are intertwined as homeostatic regulatory devices, namely [1] the progressive homeostatic integration of physiological interoceptive experiences, regarding body signals and feelings of emotion, on right anterior insula - both on 'somatic marker hypothesis' (Damásio, 1994, 2003a) and Craig's homeostatic model (Craig, 2002, 2008); [2] that happiness, fear, anger and sadness generate different activity on brain structures (Damásio et al., 2000; Lane et al., 1997), as well as specific autonomic and psychophysiological reactions (Friedman, 2010; Kreibig, 2010; Rainville et al., 2006; LeDoux, 1996), and; [3] the regulation of emotion by cognitive reappraisal and biofeedback techniques (Oschner \& Gross, 2007). As a result, mind, body and brain interact in emotional experience which, on its turn, may determine disease proneness vulnerabilities and health outcomes. Similarly, the Heidelberg Model (Greten, 2004), based on a regulatory theory and on a Leibniz's pragmatic mathematical model of systems biology, theorizes TCM as a neurovegetative functional medicine. It emphasizes also the role of emotionality on ethiopathogenics, by specifying a typical autonomic 'sign' or marker for each of the basic emotions (fear, anger, lust, happiness and sadness) on the sense of circular function and its orb iconography. This model has been tested on emotional exhaustion, depersonalization, and anxiety successfully, via traditional vegetative biofeedback exercises (e.g., Saganha, 2012; Sousa, 2012). The Heidelberg Model is therefore susceptible to be a complementary model to the Western homeostatic models of selfregulation.

Conclusion: The integration of East-West medicine concepts is a necessary step to improve scientific investigation on TCM at western healthcare systems. The neuroscience findings empirically support the Jamesian notion of 'cortical readout' feedback associated with the specificity of each emotion, a notion that is also shared by western TCMbased models and; the mechanisms underlying psychosomatic and stress-related disorder may result from bioemotional homeostatic mechanisms imbalance.

\section{Milk Production in Cows Is Improved by Moxibustion - Results from a Preliminary Study \\ Karla Pinto ${ }^{1}$, Maria João Santos ${ }^{2}$, Nuno Correia ${ }^{4}$ Jorge Machado², Henry Greten ${ }^{2,3}$ \\ ${ }^{1}$ Veterinary Medicine, Master in TCM, Abel Salazar Institute for Biomedical Sciences, Porto, Portugal; ${ }^{2}$ TCM Master Programme, Abel Salazar Institute for Biomedical Sciences, University of Porto, Porto, Portugal; ${ }^{3}$ Heidelberg School of Chinese Medicine, Heidelberg, Germany; ${ }^{4}$ Acupuncture Clinic, Alfena Private Hospital, Porto, Portugal}

Background: Moxibustion is a technique of Traditional Chinese Medicine where acupuncture points are heated by burning an herb, Artemisia vulgaris, directly or indirectly on the skin. The warmth and essence of this herb warms the $Q i$ 
and Xue in the conduits so that there is an increased in the flow of these. Moxibustion also invigorates the Yang and disperses Algor and Humor eliminating also some forms of toxins. There are two methods of therapy with moxibustion: direct moxibustion, where the moxa is placed directly on the skin, but is a rarely used technique in animals due to the scar that forms and for being a painful method; the indirect moxibustion, is the most used in animals and also in humans, where moxa rods are used at a distance of 1 to $2.5 \mathrm{~cm}$ from the acupoint to be treated or through moxa cones or balls that are placed on a insulating medium (ginger, garlic, miso, salt, vaseline, aloe gel, etc.). The time of treatment goes from 3 to 15 minutes. In Japan, moxibustion has been used to treat various diseases, such as reproductive, musculoskeletal, digestive up to urinary problems. In terms of milk production, this technique seems to promote the homeostasis hormone, stimulating the secretion of hormones when their levels are low and retaining them when they are high, leading to a perfect physiological equilibrium.

Objectives: This study aims to determine the increase in milk production through the use of moxibustion.

Methods: Ten high yield (Holstein-Friesian cows) animals were chosen and these were divided into two groups, one for intervention and the other for control ( 5 animals each). The intervention was performed for two weeks at the rate of two times per week. We used the chi-square test with probability $\mathrm{p}<0.05$, in only one of the animals in the intervention group, this variable was found.

Results: It was observed that intervened animals increased production, which makes this technique an asset and may be combined with conventional techniques.

Conclusions: This preliminary study suggested that moxibustion may be an additional technique that may improve milk production. More studies are needed to confirm this results. In addition, this study may be seen as a pionner example of integration of TCM in Veterinary Medicine in Portugal.

\section{Heidelberg Scalp Acupuncture May Improve Obstructive Sleep Apnea - A Clinical Pre-Study}

\author{
Maria João Santos ${ }^{1}$, Nuno Correia ${ }^{1,2,3,4,10}$, \\ Thomas Efferth ${ }^{5}$, Julia Vent ${ }^{6}$, Frank Brazkiewicz', \\ Jorge Machado ${ }^{1,7}$, Márcia Sá ${ }^{8}$, Christoph Doenitz ${ }^{2}$, \\ João Carlos Winck ${ }^{9}$, Henry Johannes Greten ${ }^{1,2}$
}

${ }^{1}$ Abel Salazar Institute for Biomedical Sciences (ICBAS), University of Porto, Porto, Portugal; ${ }^{2}$ Heidelberg School of Chinese Medicine, Heidelberg, Germany; ${ }^{3}$ Internal Medicine, Emergency Department, University Hospital of São João, Porto, Portugal; ${ }^{4}$ Nursing School of Oporto, University of Porto, Porto, Portugal; ${ }^{5}$ Department of Pharmaceutical Biology, Institute of Pharmacy and Biochemistry, University of Mainz, Germany; ${ }^{6}$ ENT Department of University Medicine Mannheim, Heidelberg University, Mannheim, Germany; ${ }^{7}$ Porto Biomechanics Laboratory (LABIOMEP), University of Porto, Porto, Portugal; ${ }^{8}$ Health Family Unit 'Saúde em Família' - ACeS Grande Porto IV - Maia/Valongo, Portugal; ${ }^{9}$ Lung Function and Ventilation Unit, Pulmonology Department, University Hospital of São João, Faculty of Medicine, Porto, Portugal; ${ }^{10}$ Acupuncture Clinic, Alfena Private Hospital, Porto, Portugal

Introduction: Continuous positive airway pressure (cPAP) is widely considered the 'gold standard' in Obstructive Sleep Apnea (OSA). However, two clinical studies reported a possible positive effect of conventional body acupuncture in this condition. A potential obstacle for this therapy may be the fact that 19 body acupoints had to be treated for 10 weeks to obtain lasting effects. We were interested in possible clinical effects of a new type of scalp acupuncture on OSA, which allows the usage of implantable permanent needles in one single session.

Methods: $\mathrm{N}=14$ patients met the inclusion/exclusion criteria (i.e. AHI $>5$, age 18-70, written consent, no usage of cPAP) and received acupuncture microneedles in specific points on the head according to the Heidelberg Scalp Acupuncture (HSA) model. On day 1, a baseline home cardiorespiratory polygraphy was performed to evaluate the AHI. A second polygraphy measurement was performed on day 2 or 3 after receiving HSA on the same day.

Results: The overall improvement of AHI was $51.4 \%$ ( $\mathrm{p}=$ 0.0596). Respiratory events improved by $51.3 \%$, Apnea Index by $39.4 \%$, Hypopnea Index by $73.6 \%$, oxygen desaturation by $35.9 \%$ (no statistical significance). Tendentially, patients with moderate OSA showed better improvements. No negative side effects of needling were observed.

Conclusions: These preliminary results suggest that a prospective RCT may be planned to investigate the value of HSA on the basis of our preliminary data. This should focus on moderate OSA cases and should include 33 patients in verum and control group in a parallel study design based on the calculated sample size. 


\section{Qigong Reduces Burn-Out Symptoms in Nurses-Preliminary Data from a Randomized, Interventional, Controlled Pre-Study}

\author{
António Seiça ${ }^{1}$, Nuno Correia ${ }^{3,4}, 5$, Jorge Machado', \\ Henry J. Greten ${ }^{1,2}$ \\ ${ }^{1}$ TCM Master Programme, Abel Salazar Institute \\ for Biomedical Sciences, University of Porto, Porto, \\ Portugal; ${ }^{2}$ Heidelberg School of Chinese Medicine, \\ Heidelberg, Germany; ${ }^{3}$ Internal Medicine, Emergency \\ Department, University Hospital of São João, Porto, \\ Portugal; ${ }^{4}$ Nursing School of Oporto, University of \\ Porto; Porto, Portugal; ${ }^{5}$ Acupuncture Clinic, Alfena \\ Private Hospital, Porto, Portugal
}

Background: Burn-out syndrome is an high prevalent condition in health care workers, with significant impact on their performance and with a high degree of absenteeism. Qigong, a biofeedback therapy, seems to induce a stress reduction state. To our knowledge no studies have been performed addressing Qigong effects for burn-out in health care workers.

Aim: We aimed to evaluate the influence of Qigong in nurses with burnout syndrome in a major hospital center.

Methods:

Study Design: We performed a prospective, randomized, interventional, controlled pre-study, after Hospital Ethics Committee approval. Matched pairs and random allocation to the intervention group was performed by an independent centre. Sample: Nurses from several departments were recruited $(n=44)$ and randomized into an experimental group $(\mathrm{n}=22)$ and control group $(\mathrm{n}=22)$.

Primary Outcomes: Maslach Burnout Inventory - MBI evaluated at baseline, after Qigong therapy and after summer break vacation period to assess durability of Qigong effect.

Intervention: Treatments of Qigong, after an introductory lesson, 20 minutes in the first week, and 5 minutes in the other three weeks (total: 8 sessions). In addition, subjects performed Self-Qigong twice daily for 5 minutes during 4 weeks;

Assessment (1) the experimental group, four weeks after the terminus of Qigong sessions;

Assessment (2) the control group, four weeks after the initial phase; four weeks exercise ad libitum;

Assessment (3) the control group, four weeks after the end of the study; with no controlled exrcise.

Results: Before Qigong, 68.2\% (15) of the subjects on the experimental group had a high level of burnout and $22.7 \%$ (5) had moderate level of burnout; in the control group 50.0\% (11) had a high level of burnout and 31.8\% (7) had a moderate level. There were no statistically differences at baseline burnout levels between groups $(p=0.474)$.

After Qigong, 65.0\% (13) of the experimental group showed moderate level of burnout (statistically significant in relation with the control group).

The effect of the Qigong therapy on burnout levels on the experimental group tended to persist over time.
A tendency of improvement for depersonalization and emotional exhaustion was observed; this was statistically significant $(\mathrm{p}=0.102)$ for depersonalization and not for emotional exhaustion $(p=0.042)$.

Conclusion: This pre-study showed a feasible Qigong study protocol. Results suggest that Qigong may be an effective non-invasive therapy for the treatment of burn-out in nurses. A long-term and larger study is needed to clarify this preliminary data.

\section{Acupuncture Reduction of Anxiety in Anxious Volunteers as Objectified by the Bispectral Index: Results from a Pilot Study}

Nuno Silva ${ }^{1}$, Nuno Correia ${ }^{3,4,5}$, Ruben Coelho ${ }^{1}$, Nuno Liça1 , Pedro Amorim ${ }^{1,2}$, Henry J. Greten ${ }^{2,3}$

${ }^{1}$ Psychiatry Department, Hospital Center Vila Nova de Gaia/Espinho, Vila Nova de Gaia, Portugal; ${ }^{2}$ Heidelberg School of Chinese Medicine, Heidelberg, Germany; ${ }^{3}$ Internal Medicine, Emergency Department, University Hospital of São João, Porto, Portugal; ${ }^{4}$ Nursing School of Oporto, University of Porto; Porto, Portugal; ${ }^{5}$ Acupuncture Clinic, Alfena Private Hospital, Porto, Portugal; ${ }^{6}$ Anesthesiology Department, Centro Hospitalar do Porto, Porto, Portugal; ${ }^{7}$ Plastic Surgery, Coimbra Hospital Center; ${ }^{8}$ Family Medicine, Health Center, Algarve

Background: Acupuncture studies on anxiety using bispectral index of the EEG (BIS) as a measure of anxiolysis have shown conflicting results.

Aims: To assess electroacupuncture (EA) effects on anxiety as measured by the BIS.

Methods: 15 anxious volunteers were recruited for a prospective, controlled, blinded, crossover study with random group allocation. Low-frequency EA was performed on $\mathrm{He} 7$ and on control point Lu 9 (Lung 9) in separated sessions. Outcomes: Anxiety measured by visual analogue scale; BIS data at rest state (10 min), during EA (20 min), and post EA (5 min).

Results: Acupuncture significantly decreased selfassessed anxiety levels on the control and experimental group $(p=0.002$ and $p=0.001)$; with no in-between group differences. Acupuncture on He7 didn't show significant decrease in BIS compared to the rest state $(89.7 \pm 5.0$ to $86.5 \pm 7.2 ; \mathrm{p}>$ $0.05)$. However, acupuncture on $\mathrm{He} 7$ was related to a significant decrease in BIS as compared to Lu9 (means: $90.7 \pm 4.3$ to $86.5 \pm 7.2 ; \mathrm{p}=0.009$. Minimum BIS was also significantly lower for He7 (80.6 \pm 9.6 to $71.9 \pm 12.3 ; p=0.011$ ).

Conclusion: Acupuncture on $\mathrm{He} 7$ was superior to $\mathrm{Lu} 9$ in decreasing BIS values in anxious volunteers but it didn't show BIS reduction compared to the rest state. Self-perceived anxiety decreased with acupuncture on both groups. However EA on He7 wasn't superior to EA on Lu9 in decreasing anxiety. We conclude that He7 has a measurable effect on the BIS compared to Lu9 suggesting a neurological specific pathway. 


\section{Qigong Reduces Performance Related Anxiety and Physiological Stress Functions in Transverse Flute Music School Children as Objectified by Cortisol and Heart Rate Measurements}

\author{
Cláudia Maria Sousa1, Mário Goncalves ${ }^{1,2,3}$, \\ Jorge Machado1, Thomas Efferth ${ }^{4}$, Tobias Greten ${ }^{2}$, \\ Petra Froeschen ${ }^{2}$, Henry J. Greten 1,2,3 \\ ${ }^{1}$ Institute of Biomedical SciencesAbel Salazar, University \\ of Porto, 4099-030 Porto, Portugal; ${ }^{2}$ German Society \\ of Traditional Chinese Medicine, 69126 Heidelberg, \\ Germany; ${ }^{3}$ Heidelberg School of Chinese Medicine, \\ 69126 Heidelberg, Germany; ${ }^{4}$ Department of \\ Pharmaceutical Biology, Institute of Pharmacy and \\ Biochemistry, University of Mainz, 55099 Mainz, \\ Germany
}

Background: Anxiety disorders are common psychiatric conditions that can cause significant disability, poor quality of life and enormous social costs. Anxiety and improvement of learning are closely related. Vegetative changes as seen in stress syndrome may also impair functional abilities of the patients. This is frequently seen in musicians who require an ultimate motor and breathe control. In flutists performancerelated anxiety may potentially cause problems that empirically include anxiety, heartbeat, blood pressure, muscular tension and cold hands. This study aims to evaluate if and how Qigong-related effects may be further objectified by physical measurable parameters and psychological scores.

Methods: $\mathrm{n}=1610$ to 12 year old music school children playing the transverse flute were divided into control $(n=8)$ and Qigong $(n=8)$ group. In the Qigong group musicians received Qigong lessons of the so-called white ball over 7 weeks, twice a week, for 30 minutes and they were instructed to do the exercises at home daily. In the control group children didn't receive any intervention (waiting list design). Heart rate, blood pressure, surface electromyography, time reaction, salivary cortisol level and subjective perception of anxiety measured by EADS-C were measured before and after intervention.

Results: Qigong reduced subjective perception of anxiety (mean of 4.5); salivary cortisol levels (mean of $0.198 \mu \mathrm{g} / \mathrm{dl}$ ); heart rate (mean of 11 beats per minute); blood pressure (6.54 mm Hg). However, only heart rate decrease showed a significant change ( $\mathrm{p}$ value $=0.005$ ). There is no evidence that Qigong is effective in the reduction of muscular tension of trapezius (measured by SEMG) and in the reduction of time reaction.

Conclusion: Qigong effects can be measured properly by psychological scores and physiological parameters such as salivary cortisol and heart rate. The obvious effects of the white ball Qigong on performance related anxiety and related physical disorders was measured in this study.

\section{Acupuncture May Improve Work-Related Musculo-Skeletal Disorders in Professional Orchestra Musicians}

Cláudia Maria Sousa ${ }^{1}$, Jorge Machado', Daniela Coimbra², Manuel Lopes-Lima1, Thomas Efferth ${ }^{5}$, Susana Seca ${ }^{1}$, Mário Goncalves 1,3,4, Henry J. Greten ${ }^{1,3,4}$

${ }^{1}$ Institute of Biomedical SciencesAbel Salazar, University of Porto, 4099-030 Porto, Portugal; ${ }^{2}$ Superior School of Music and SpectacleArts, University of Porto, 4099-030 Porto, Portugal; ${ }^{3}$ German Society of Traditional Chinese Medicine, 69126 Heidelberg, Germany; ${ }^{4}$ Heidelberg School of Chinese Medicine, 69126 Heidelberg, Germany; ${ }^{5}$ Department of Pharmaceutical Biology, Institute of Pharmacy and Biochemistry, University of Mainz, 55099 Mainz, Germany

Background: Many Traditional Chinese Medicine (TCM) practitioners believe that the right TCM diagnosis is the basis of optimal clinical results of acupuncture. However, establishing the diagnosis in TCM by pulse diagnosis, tongue diagnosis and clinical signs is a time consuming and difficult task, requiring years of experience and clinical training. Palpation of acupoints may support this process since the classics, as seen in the comparison of pressure-sensitivity of skin areas such as mu-points and ashi points.

Methods: A prospective, randomized, controlled, single blinded clinical trial (RCT) was performed in $\mathrm{n}=41$ professional orchestra musicians with Work Related Musculoskeletal Disorders (WRMSD). Pressure sensitivity was assessed on 24 acupoints on each side of the body and pressure sensitivity systematically ranked and interpreted according to an interpretation matrix for acupoint selection. Pain was treated by compression (An) of real acupoints in the intervention group and of non-specific skin points on the thigh in the control group in the same manner and intensity. Individual pain reduction was assessed by the mean reduction in VNS of all pain spots per individual.

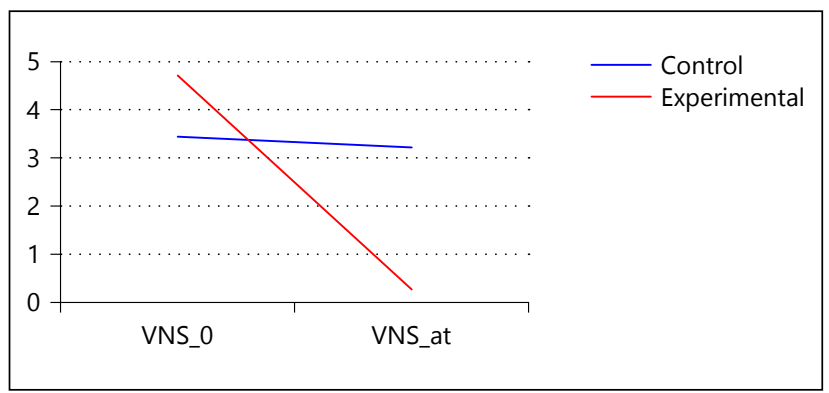

Fig. 1. Before intervention (VNS-0), pain was comparable in both groups, 15 min after intervention (VNS-at), pain decreased significantly more in the experimental group than in controls. 
Results: Both groups revealed comparable pain intensity before treatment $(p=0.136)$. After the first treatment pain was reduced in the experimental group by an average of $95.5 \%(p=0.00)$ from mean VNS 4.4 to mean VNS 0.2. In the controls, however, pain reduction was only $8.8 \%(\mathrm{p}=0.034)$ from mean VNS 3.4 to mean VNS 3.1 revealing astatically relevant difference after treatment $(\mathrm{p}=0.000)$ between the groups (See figure 1).

Conclusion: Acupoint selection based on systematic palpation of acupoints leads to a considerable success rate in the treatment of WRMSD and may serve as a self-treatment method in Orchestra musicians and other groups of employees with repetitive movements and psychological stress resulting in WRMSD.

\section{Acupuncture Improves Vertical Jump Performance in Volleyball Players - Results from a Pilot Study}

\author{
Nuno Sousa ${ }^{1}$, Nuno Correia 1,4,5,6, Alexandre Medeiros², \\ Jorge Machado ${ }^{1,7}$, Manuel Laranjeira ${ }^{1}$, Henry J. Greten ${ }^{1,3}$ \\ ${ }^{1}$ Abel Salazar Institute for Biomedical Sciences, \\ University of Porto, Porto, Portugal; ${ }^{2}$ Faculty of Sports, \\ University of Porto, Porto, Portugal; ${ }^{3}$ Heidelberg \\ School of Chinese Medicine, Heidelberg, Germany; \\ ${ }^{4}$ Internal Medicine, Emergency Department, University \\ Hospital of São João, Porto, Portugal; ${ }^{5}$ Nursing \\ School of Oporto, University of Porto, Porto, Portugal; \\ ${ }^{6}$ Acupuncture Clinic, Alfena Private Hospital, Porto, \\ Portugal; ${ }^{7}$ Porto Biomechanics Laboratory (LABIOMEP), \\ University of Porto, Porto, Portugal
}

Background: The vertical jump skill is the most significant physical movement to be trained in volleyball. Previous studies in health athletes suggest that acupuncture may improve physical performance including increased muscle strength.

Objective: The aim of this study was to determine whether there is an immediate effect of acupuncture on the performance of vertical jump in professional volleyball players.

Methods: 26 male athletes of high competition (age, weight, years of practice and height of $24.31 \pm 1.28$ years, $82.27 \pm 1.89 \mathrm{~kg}, 13.38 \pm 1.28$ years and $187.00 \pm 1.34 \mathrm{~cm}$, respectively) placed in National Division A1 with a minimum of 4 years in competition were included.

Exclusion Criteria: Injury or pain at lower legs or another region that could compromise the intended performance, alcohol consumption, sedatives or pain drugs over past 48 hours; present balance disorders or any neurological impairment; interruption of activity over 6 months.

Design: Athletes were randomly assigned to a control group ( $\mathrm{n}=10)$ and intervention group $(\mathrm{n}=16)$. The intervention group received stimulation of the S34, whereas in control group a non-acupuncture point located 2 thumbs from the interventional point between the bladder and gallbladder conduits was used. The mean of 3 jumps prior and after acupuncture were compared by Wilcoxon test.

Results: In the intervention group we found an increase of $0.94 \mathrm{~cm}$ (significant difference $(\mathrm{p}<0.05)$. In the control group we found no statistically significance difference with a mild tendency to lower jump heights. Comparison of the two groups showed a significant difference $(p<0.05)$ as the intervention group increased jumping power whereas in the CG the jumping power decreased slightly ( $p>0.05)$.

Conclusion: This preliminary study shows that acupuncture is able to induce an increase in vertical jumping in high level volleyball players, supporting the need for further randomized double-blinded controlled trials.

\section{Ear Acupuncture May Lower Anxiety on University Students: A Neurovegetative Assessment by Evaluating the Status of Tongue Changes}

Andreia Vieira ${ }^{1}$, Jürgen Stürmer ${ }^{1}$, Karine Silva $^{7}$, Maria João Santos ${ }^{1}$, Mariana Hinzmann ${ }^{1}$, Nuno Correia ${ }^{3,4,5}$, Jorge Machado ${ }^{1}$, Henry J. Greten ${ }^{1,2,6}$

${ }^{1} \mathrm{TCM}$ Master Programme, Abel Salazar Institute for Biomedical Sciences, University of Porto, Porto, Portugal; ${ }^{2}$ Heidelberg School of Chinese Medicine, Heidelberg, Germany; ${ }^{3}$ Internal Medicine, Emergency Department, University Hospital of São João, Porto, Portugal; ${ }^{4}$ Nursing School of Oporto, University of Porto; Porto, Portugal; ${ }^{5}$ Acupuncture Clinic, Alfena Private Hospital, Porto, Portugal; ${ }^{6}$ Deutsche Gesellschaft für Traditionelle Chinesische Medizin, Heidelberg, Germany; ${ }^{7}$ Abel Salazar Institute for Biomedical Sciences, University of Porto, Porto, Portugal

Introduction: High-level students are exposed to various challenges that trigger high anxiety levels in relation to their academic requirements. This study aims at evaluating a potential effect of auricular acupuncture (AA) on the anxietylevels of university students.

Methods: After a pre-study, an experimental, prospective, randomized,controlled and single-blinded study was conducted. The sample included university students $(n=69)$, naïve to acupuncture, under no psychotropic medication, and with no known psychiatric or thyroid disorders. Individuals were randomly allocated to the Experimental group (EG, $n=25)$ : verum AA in points; the Placebo group ( $P G, n=22)$ : sham $A A$ on points in neurological segments distinct from the verum $A A$ and the waitling list group ( $\mathrm{WL}, \mathrm{n}=22)$ : without any AA treatment. One week prior to the examination series at the University, subjects were treated with AA using semi-permanent needles in place for 48 hours. maAssessments were made 5 minutes before (T0) and 48 hours after (T1) the needling.

Main Parameters: Level of anxiety was assessed according to the state anxiety inventory STAI (form Y1), a 
Visual Analogue Scale (VAS) for anxiety, a neurovegetative status by a scored analysis of the tongue according to the principles of Traditional Chinese Medicine (NS-TCM), Blood pressure, heart and respiratory rate.

Results: 69 students ( 49 women; 20 men) were included, with (age $=20.8+4.71$ years, weight $=62.1+9.16 \mathrm{~kg}$, height $=$ $1.69+0.07 \mathrm{~m}$ and body mass index $=21.73+2.25 \mathrm{~kg} / \mathrm{m}^{2}$ ), $15.5 \%$ showed moderate to severe states of anxiety and $12 \%$ trace of anxiety. In the EG, a significant reduction of the anxiety levels was observed, according to STAI-form Y1 ( $p=0.031)$, VAS ( $\mathrm{p} \leq 0.01)$ and the NS-TCM ( $\mathrm{p} \leq 0.01)$. No significant differences were found on the other measurements and both the PG and WL.

Conclusion: This study suggests that AA is effective in reducing exam-related anxiety in university-level students, as corroborated by an improvement on the NS-TCM.

\section{Acupuncture Effects on Microcirculation in Patients with Proximal Femur Fracture: Development of Objective Assessment a Step Toward Scientific Validation of TCM}

\author{
Ana Anjos 1,3,4, Christoph A. Donitz 1,2,3, Thomas Efferth ${ }^{5}$, \\ Tobias Greten ${ }^{2,3}$, Henry J. Greten ${ }^{1,2,3}$ \\ ${ }^{1}$ Institute of Biomedical Sciences (ICBAS), University \\ of Porto, Porto, Portugal; ${ }^{2}$ German Society of \\ Traditional Chinese Medicine, Heidelberg, Germany; \\ ${ }^{3}$ Heidelberg School of Chinese Medicine, Heidelberg, \\ Germany; ${ }^{4}$ Institute for Neurosciences, Porto, Portugal; \\ ${ }^{5}$ Department of Pharmaceutical Biology, Institute of \\ Pharmacy and Biochemistry, University of Mainz, Mainz, \\ Germany
}

Objective: The aim of this study was to demonstrate whether ir is possible to objectively assess the effects of acupuncture by microcirculation-related parameters in the given clinical scenario, to evaluate the significance of the status of capillary perfusion prior to acupuncture for the treatment of a clinical cold pattern, and to evaluate the possible role of microcirculation-related parameters for the futures parameterization of the traditional Chinese medicine (TCM) diagnosis.

Methods: This prospective, uncontrolled, unblinded preliminary clinical trial included 32 elderly patients after surgical treatment for femoral fractures. For acupuncture treatment the patients received acupuncture with the 'leopard spot technique' on the stomach meridian (Liangqiu, S34/ ST34). Measurements of microcirculation-related parameters (velocity, blood flow, haemoglobin, oxygen saturation) by white light spectroscopy and laser Doppler were performed prior to and after acupuncture treatment.

Results: Two subgroups of patients after femur fracture could be identified: a low- and a high-perfusion group. Capillary flow velocity and blood flow were significantly augmented by acupuncture in the low-perfusion group only.
In the high-perfusion group, there was no significant change of perfusion after acupuncture. The statistical analysis of all patient regardless of their pre-acupuncture perfusion status revealed no statistically significant alteration after acupuncture. The acupuncture effect may have been masked by the inhomogeneity of the overall group.

Conclusion: Microcirculation-related parameters may be valuable to measure acupuncture effects objectively and to characterize the vegetative functions prior to acupuncture so as to homogenize the comparison groups in clinical trials. In our example, a local cold pattern (low capillary prefusion of the leg) could be treated successfully by a pint which enhances qi and blood flow, whereas in heat pattern (high capillary perfusion) this intervention had no such effect. Future studies may be directed to correlated the vegetative status as measurable by TCM vegetative parameters with the key symptoms of TCM diagnosis.

\section{Acupuncture in the Treatment of Plantar Heel Pain: A Clinical Research Protocol}

\author{
Fernando Barros ${ }^{1}$, Nuno Correia ${ }^{2,5}$, Henry J. Greten ${ }^{3,4,5}$ \\ ${ }^{1}$ Master Programme of TCM - Abel Salazar Institute \\ for Biomedical Sciences, Porto, Portugal; ${ }^{2}$ Acupuncture \\ Clinic, Alfena Private Hospital, Porto, Portugal; ${ }^{3}$ German \\ Society of Traditional Chinese Medicine, 69126 \\ Heidelberg, Germany; ${ }^{4}$ Heidelberg School of Chinese \\ Medicine, 69126 Heidelberg, Germany; ${ }^{5}$ Internal \\ Medicine, Emergency Department, Hospital Center São \\ João, Porto, Portugal
}

Introduction: Plantar heel pain (PHP) is one of the most common musculoskeletal pathologies of the foot. It affects about $10 \%$ of the population at least once throughout their lives. Common therapies, like foot pads, physical therapy, NSAID's, cortisone injections, and electroshock waves, are regarded as with limited impact on prognosis. Acupuncture in PHP is widespread in Asia but only two studies are available in peer reviewed Western journals, which are inconclusive and enclose methodological problems such as criteria underlying point selection and lack of objective measurements, such as by pressure-algometry.

Objectives: (1) to compare effects of TCM-based acupoint selection versus effects of points out of the known conduit system; (2) to compare needling with laser acupuncture so as to find the optimal stimulation parameters.

Methods:

Design: Prospective, randomized, single blinded and controlled study, as already approved by the respective departments of the Hospital São Sebastião - Entre Douro e Vouga Hospital Center, and submitted to the Ethical Committee.

Recruitment: A sample of 60 patients, with diagnosis of plantar heel pain when standing, will be voluntarily recruited from the Department of Orthopedics. Eligibility evaluation in the Anesthesiology Department, obtainment of informed 
consent and are randomized in to two thirty elements groups. From the first thirty, ten will be selected to compare VA to CA with five elements each.

Intervention: The verum acupuncture group (VA) will be treated with R7, V66, and L6, by the leopard spot technique; the control group (CA) will be acupunctured on three points in skin areas located at least in $3 \mathrm{~cm}$ distance to the above mentioned acupoints, but out of the conduit system. Laser acupuncture group (LA) with Laser Needle device and treatment of the same 14 VA group points using a wavelength of $685 \mathrm{~nm}$, power density of $4.6 \mathrm{~kJ} / \mathrm{cm}^{2}$ per point, during 20 min. Pressure algometry will be compared before and directly after one single needling treatment to measure acute effects by pressure tolerance between VA and CA group. In a second trial, the points of the group with the best result will be treated by conventional needling vs. Laser acupuncture to find out about the optimal stimulation parameters twice a week over three weeks. Main parameters will be measured before and after each treatment and include: visual analogue scale, pressure algometry (using validated pedography platform) and a simple regular scale. After treatment completion all subjects will be recalled after 3 weeks for follow-up measurements.

Results: Significant differences between the VA and CA group are expected. We hypothesize by practical experience, that the treatment with the best acute effects will probably also be better working in chronic treatment. Comparison between VA and LA may reveal some more information about the optimal type of stimulation.

Conclusions: The study is designed to reveal basic data for a prospective randomized double-blinded study in the future with optimal stimulation parameters and point selection, and objective measurements by pressure algometry in addition to VAS.

\section{Microcirculation in Raynaud Phenomenon May Be Augmented by Qigong as Objectified by Thermography - Results from a Clinical Pre-Study}

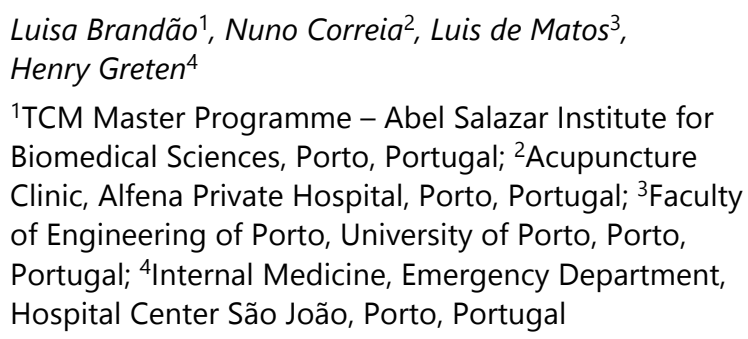
Biomedical Sciences, Porto, Portugal; ${ }^{2}$ Acupuncture Clinic, Alfena Private Hospital, Porto, Portugal; ${ }^{3}$ Faculty of Engineering of Porto, University of Porto, Porto, Portugal; ${ }^{4}$ Internal Medicine, Emergency Department, Hospital Center São João, Porto, Portugal

Background: Raynaud's Phenomenon (RP) is a common clinical sign, with a prevalence of 3-5\% in the general population, predominantly in females, the majority (90\%) being primary. It is characterized by a vasospastic reaction of the extremities (hands and feet), leading to episodes of digital ischemia, caused by exposure to cold or stress. Previous studies show a massive enhancement of microcirculation induced by Qigong 'white ball', we were interested whether it may be useful to treat RP. In order to develop a working and a measurement system for study clinical pre-trial, nonrandomized, controlled, unblinded, the first experiments were conducted for future investigations.

Objectives: 1 . To evaluate microcirculation in patients with Raynaud phenomenon by thermography, 2 . Check the effect of Qigong in patients without Raynaud's Phenomenon, with primary RP and secondary RP, and 3 . To gain experience for the development of new studies.

Methods: According to the set of inclusion and exclusion criteria, 13 individuals were recruited for a preliminary study and allocated in three groups of patients without RP; with primary RP, and with secondary RP associated to rheumatic disease. The temperature was measured by infrared thermography on the tip of the middle finger (TMF) and the point Pericardium 8 (PC8) before and after Qigong 'White Ball', at baseline and after completion of cold stimulation test (CST).

Results: Due to the small sample, these results are not significant, since there were no statistically significant differences.

1. Thermography in patients with and without RP was possible and relatively easy to do.

2. At the tip of the middle finger without cold stimulation test revealed that, patients without FR show marked enhancement of microcirculation, primary RP patients show less significant, but clear enhancement of microcirculation, secondary RP patients showed smaller increases microcirculation, but he still existed.

3. The temperature increased in TMF and PC8 was lower in RP secondary compared to the FR primary, and the magnitude of the increase in RP in two groups with the cold stimulation test was superior to the baseline.

Discussion: The improvement in microcirculation was superior in the group without RP in comparison with the Raynaud group and the improvement in the secondary Raynaud was smaller than the primary. Although not statistically significant, there was an increase in temperature in both types of Raynaud in TMF compared to baseline, suggesting a possible effect of Qigong. It is believed that primary RP is a neurovegetative phenomenon, and can be considered that secondary RP involves also changes in the humoral immune system. This may be a cause for differences in microcirculation measured by thermography.

Conclusion: Microcirculation in Raynaud phenomenon may improve with Qigong. Further study with larger samples (i.e. 30/30/30) may be a promising project, as well as further trials comparing Raynaud Phenomenon with and without Qigong therapy. 


\section{Outcomes from a Traditional Chinese Medicine Master Programme in a Biomedical Science Faculty at Porto University - A Scientific Educational Model}

\author{
Nuno Correia ${ }^{1,4,6}$, Maria João Santos 1 , \\ Jorge Machado ${ }^{1,5}$, Henry J. Greten 1,2,3 \\ ${ }^{1}$ TCM Master Programme, Abel Salazar Institute for \\ Biomedical Sciences (ICBAS), University of Porto, Porto, \\ Portugal; ${ }^{2}$ Heidelberg School of Chinese Medicine, \\ Heidelberg, Germany; ${ }^{3}$ Deutsche Gesellschaft für \\ Traditionelle Chinesische Medizin, Heidelberg, \\ Germany; ${ }^{4}$ Internal Medicine, Emergency Department, \\ University Hospital of São João, Porto, Portugal; ${ }^{5}$ Porto \\ Biomechanics Laboratory (LABIOMEP), University of \\ Porto, Portugal; ${ }^{6}$ Acupuncture Clinic, Alfena Private \\ Hospital, Porto, Portugal
}

Background: Traditional Chinese Medicine (TCM) is one of the oldest medical systems in the world. Currently, the Portuguese government is discussing national rules on the education and professional practice of non-conventional therapies including TCM. Lack of accredited university-level education on TCM addressed.

Objective: To reveal erience from a University Master Degree in TCM following a scientific model of TCM known as the 'Heidelberg Model' (HM).

Results: Porto University in Portugal has established the first TCM Master (2008) in a state University. The program is open to medical doctors and other health-care and lifesciences graduates. A three semester core intensive training (specialization course) is provided covering Acupuncture, Phytotherapy, Qigong, Dietetics, Psycotherapy and Tuina. An additional semester is dedicated to the Master thesis. The biomedical approach to TCM according to the HM has largely contributed to this acceptance. Postulated neurohumoral mechanisms on a regulatory model support TCM functional diagnosis and treatment strategies. Over the past 7 years, 147 students enrolled in this course and $25.5 \%$ concluded the master thesis. Around $91 \%$ of the students are non-medical doctors. On the University library, 25 theses are available on-line. Five articles have been published; 14 abstracts were accepted in scientific meetings; 1 student is on a PhD; 4 students received academic prizes.

Conclusion: This is the first TCM course to be approved by a public University in Europe. Our Master programme demonstrates that a scientific model of TCM is needed to provide rigorous education as well as to support high-level research towards the integration of TCM in modern health care.

\section{Applying a Novel Pressure-Sensitive Sensor Device to Measure the Acute Effects of Acupuncture for Pelvic Pain Due to Endometriosis: A Clinical Protocol}

\author{
K. Ibar', N. Correia ${ }^{2,3}$, M.J. Santos ${ }^{4}$, A.R. Costa ${ }^{5}$, \\ H.J. Greten ${ }^{6}$ \\ ${ }^{1}$ TCM Master Programme, Abel Salazar Institute \\ for Biomedical Sciences, University of Porto, Porto, \\ Portugal; ${ }^{2}$ Acupunture Clinic, Alfena Private Hospital, \\ Porto, Portugal; ${ }^{3}$ Internal Medicine, Emergency \\ Department, Hospital Center São João, Porto, Portugal; \\ ${ }^{4}$ Gynecology Department, Hospital Center São João, \\ Porto, Portugal; ${ }^{5}$ Heidelberg School of Chinese \\ Medicine, Heidelberg, Germany
}

Introduction: Endometriosis (EM) is a common health problem affecting women worldwide and often underdiagnosed. In western medicine, treatment of EM needs life-long management including medical or surgical treatments, being hormonal replacement therapy the most used. Long term use of these therapies remains challenging due to the plethora of serious adverse effects. Few studies have been published on acupuncture for 'Endometrialgia'. Acupuncture has been studied in Gynecological disorders but its effectiveness for pain in endometriosis remains uncertain. The use of acupuncture may be benefitial in relieving the chronic pelvic pain which in turn improves patient's quality of life.

\section{Objectives:}

1. To develop a clinical research protocol for a randomized, sham-controlled preliminary study that evaluates the acute effects of acupuncture for endometriosis related pain.

2. To objectively measure the effect of acupuncture in pain, by using a pressure sensitive sensor device.

3. To assess feasibility of the study protocol, and collect preliminary data, for a larger study.

Methods:

Design: A clinical protocol of preliminary, prospective, randomized, controlled, single-blinded clinical trial design. Women age 18-55. The acupuncture is manual Blood-letting technique on GB41, and Sp6.

Setting: Sao Joao Hospital, gynecology department EM clinic. The protocol is approved by the ethical Committee of Centro Hospitalar de Sao Joao.

Sample: 30 patients, diagnosed with pelvic Endometriosis, and have Pelvic pain secondary to EM. Randomly assigned into two groups by coin flip into Experiment and Control group. All patients consent obtained before the beginning of the intervention.

$\checkmark$ Experiment group: 15 patients will undergo true acupuncture in points chosen according to HM of TCM.

$\checkmark$ Control group: 15 patients will undergo sham acupuncture in non-acupoints 1 Cun distant from the Acu-points. 


\section{Eligibility:}

Inclusion Criteria: All women with pelvic pain due to EM at Sao Joao Hospital EM clinic, mentally fit and co-operative with the study.

Exclusion Criteria: Women with menses (to exclude dysmenorrhea), chronic pelvic pain due to non-endometriosis causes, active infectious disease, congenital diseases or any other severe disease. Patients who already tried Acupuncture for the pelvic pain.

Intervention: First step is Pain assessment using specially designed sensor for this study while applying deep pressure on the pelvic region and also visual analogue scale.

Second step is the acupuncture. The type of the acupuncture is manual acupuncture/blood-letting technique with two sessions of acupuncture once per week.

$>$ Verum (true) acupuncture will be performed in selected points according to Heidelberg model of TCM: GB41, SP6.

- False acupuncture will consist of non-specific acupuncture: false skin points 1 Cun adjacent to verum acupoints.

Final step is the crossover of the two groups after one week of wash out.

Results: The effect of acupuncture as analgesic is demonstrated in the literature. Recruitment for this study has recently started at Hospital São João, a major central university hospital in Porto. The study may reveal that a significant relief could be achieved after the application of acupuncture in patients with chronic pelvic pain due to endometriosis. This research can be used as step towards the possible inclusion of acupuncture in the guidelines for the management of EM induced pelvic pain.

Discussion: A novel pressure-sensitive sensor device was specially developed which allows objective measurement of acupuncture's effect in pain in endometriosis patients. Results from this study may in the future grant a larger prospective, controlled, multi-blinded, randomized protocol with longer follow-up period. Acupuncture may prove to be another interesting tool to manage this condition responsible for a great discomfort and reduced quality of life for affected women and their partners.

\section{Acute Acupuncture Treatment Improves Temporomandibular Disorder (TMD) in Shaoyang Syndrome: A Preliminary Study}

Raquel Jacinto 1,3, Vânia Barros ${ }^{2,4}$, Nuno Correia ${ }^{2,5}$, Henry Greten ${ }^{3,6}$

${ }^{1}$ Physiotherapy Department, Viana do Castelo Private Hospital, Viana do Castelo, Portugal; ${ }^{2}$ Acupuncture Clinic, Alfena Private Hospital, Porto, Portugal; ${ }^{3}$ TCM Master Programme, Abel Salazar Institute for Biomedical Sciences, Porto, Portugal; ${ }^{4}$ Operative Dentistry, Health Sciences Institute, Dental Medicine, Portuguese Catholic Universtity, Viseu, Portugal; IInternal Medicine, Emergency Department, Hospital Center São João, Porto, Portugal; ${ }^{6}$ Heidelberg School of Chinese Medicine, Heidelberg, Germany

Background: A previous systematic review suggested that acupuncture may be effective in the treatment of temporomandibular joint dysfunction (TMD). However, appropriate controls widely lacked, thus limiting the level of evidence.

Objective: To evaluate the acute effects of acupuncture on pain and movement in patients with TMD, in comparison to control acupuncture done on non-acupuncture points, but with same intensity and depth of needling.

Methods: We developed a single-blinded, randomized, controlled study design with a verum therapy concept as objectively measured by caliper and VAS in a preliminary study. After approval by the Ethical Committee, patients with TMD diagnosed by an independent dentist and with TCM diagnosis of ALT III (shaoyang Syndrome) as defined by the Heidelberg model, were recruited from a specialized Physiotherapy hospital unit. The Experimental group was subject to verum-acupuncture in points: S13 (St13), F21 (GB21), Tk5 (TB5) by the leopard spot technique. Non-acupoints in distinct dermatomes were selected for the control needling by the same technique, amount and depth as the verum group. Measurements were performed before (T0) and (T1) $5 \mathrm{~min}$ after the needling intervention through assessment by (1) VAS for myofascial, articular pain and pain with maximum mouth opening; (2) digital caliper measurements in maximal teeth occlusion, and maximal mouth opening; (3) Chronometry measure time to maximal mouth opening until pain appearance.

$\mathrm{N}=7$ patients were so far included, 4 in the experimental group and 3 in the control group, aged between 22 and 36 years-old, the experiment is still in process.

Results: Pain assessment by VSA showed statistical significant differences on standardized temporal-, masseter-, and lateral pterygoid muscle palpation and on temporal tendon palpation, as well as pain in maximum mouth opening $(p=0.03$, respectively). No improvement was shown for intercuspal position $(\mathrm{p}=0.72)$, but a positive tendency was revealed in maximal mouth opening $(\mathrm{p}=0.07)$ and in time until pain $(\mathrm{p}=0.05)$. 
Conclusions: This study showed that acupuncture according to a TCM diagnosis for TMD may reduce patient's pain perception and objective signs and symptoms of TMJ dysfunction. In addition, these data show specific effects of real acupuncture in comparison with unspecific skin needling.

\section{Reduction of Menopause-Related Symptoms by Qigong as Neurovegetative Biofeedback Therapy: A Pilot Study}

\author{
Ana Moreira ${ }^{1,4}$, Henry Greten 1,2,3 \\ ${ }^{1}$ TCM Master Programme, Abel Salazar Institute \\ for Biomedical Sciences, University of Porto, Porto, \\ Portugal; ${ }^{2}$ German Society of Traditional Chinese \\ Medicine, 69126 Heidelberg, German; ${ }^{3}$ Heidelberg \\ School of Chinese Medicine, 69126 Heidelberg, \\ Germany; ${ }^{4}$ Health Family Unit 'Viver Mais', Health \\ Center of Castelo da Maia, Maia, Portugal
}

Background: Menopausal symptoms are common among women, often causing distress, sleep deprivation and reduced quality of life. Hormone therapy (HT) has frequently been recommended for relief of hot flushes, but concerns about the health risks of HT have encouraged women to seek alternative treatments. Only one study on Qigong.

Objective: The present study was designed to assess whether Qigong can improve vegetative functions, perceptions of symptoms, emotional stability, and quality of life, on women that cannot use HRT.

Materials and Methods: Out of 68 first appointments from the menopausal department, eleven postmenopausal women, between 36 and 59 years, with menopause syndrome validated by an independent gynaecologist, contraindication to hormone replacement therapy, and written consent, received Qigong specific training during 8 weeks. Assessments were made using the Kupperman index score for clinical manifestations, Hospital Anxiety and Depression Scale and World Health Organization Quality of Life short-version scale. Salivary cortisol levels were additionally determined as an objective parameter for measuring stress.

Results: All scores improved over time, showing a significant $(\mathrm{p}<0.05)$ reduction in the Kupperman score $(44 \%)$ and depression (14\%), and also an improvement on all quality of life parameters (10\%). On a sub-scale level, positive regression of vasomotor symptoms, paraesthesia, insomnia, weakness, arthralgia and myalgia, headaches, palpitations and vertigo accomplished significance, but nervousness, melancholia and anxiety did not. Salivary cortisol levels diminished along the weeks of training (40\%), although results are not statistically significant. There was no significant correlation between psychometric parameters and cortisol levels.

Conclusions: Qigong improved perception of menopause symptoms, reduced depression and promoted quality of life, indicating this method to be a valid therapeutic option to be considered. Although the results are promising, continuation of the study with an increase of the number of cases and improved methodology is recommended.

\section{Acupuncture Increases the Elbow Extension as Objectified by the Median Neurodynamic Test: Results from a Randomised, Double-Blind, Non-Inferiority Trial}

\author{
Nuno Morais ${ }^{1}$, Henry Johannes Greten ${ }^{2,3}$, \\ Maria João Santos ${ }^{3}$ \\ ${ }^{1}$ Polytechnic Institute of Leiria, School of Health \\ Sciences (ESSLei-IPL), Leiria, Portugal; ${ }^{2}$ Heidelberg \\ School of Chinese Medicine (HSCM), Heidelberg, \\ Germany; ${ }^{3}$ University of Porto, Instituto de Ciências \\ Biomédicas Abel Salazar (ICBAS-UP), Porto, Portugal
}

Background and Purpose: Musculoskeletal pain syndromes may show signs of neural involvement, such as increased mechanossensitivity to pressure and tension of the peripheral nerves. Acupuncture has shown positive effects in people suffering from peripheral nerve dysfunction. However, whether these benefits are related to reduced mechanossensitivity when tension is applied to the peripheral neural tissues (neurodynamics) is unknown. The purpose of this study was to evaluate the acute effects of acupuncture in the response of a neurodynamic test applied to the median nerve (MNT1).

Methods: Thirty-one ( $\mathrm{n}=31$ ) asymptomatic subjects with normal posture and mobility of the upper body quadrant joints volunteered to participate. After signing the informed consent, subjects, who were blinded to the real study purpose, were randomly assigned to one of 2 groups: one group $(n=14$; $\sigma^{x}=3$; age = 34.6 \pm 11.1 years old; mass = 62.5 $\pm 14.1 \mathrm{Kg}$; height $=167.1 \pm 9.1 \mathrm{~cm}$ ) was submitted to acupuncture (leopard spot needling technique) on one classical acupoint believed to be beneficial in people with symptoms of median nerve dysfunction (Pc 5 or Jiānshǐ); the other group ( $\mathrm{n}=17$; $\sigma^{x}=3$; age $=33.6 \pm 12.6$ years old; mass $=60.5 \pm 8.5 \mathrm{Kg}$; height $=$ $167.1 \pm 10.1 \mathrm{~cm}$ ) was submitted to the same technique on a new acupoint ( $5 \mathrm{~cm}$ below P 5 or Chǐzé) selected upon the clinical experience of one of the authors (H. J. G.). One assessor, blinded to group allocation and measurement results, performed the MNT1 on the dominant arm, before and after acupuncture. On both occasions, subjects were asked to report the onset of symptoms (e.g., stretching, tingling) as the elbow was being passively moved from $90^{\circ}$ of elbow flexion to extension, with the arm at $90^{\circ}$ of shoulder abduction. The test ended when the maximum range of motion (ROM) tolerated was reached (fig. 1). Elbow extension ROM (in degrees) was assessed using a smartphone (iPhone 4, iOS 7.1.2, app Compas, Apple Inc., Cupertino CA, USA). Two 2-way repeated measures analysis of variance (ANOVA) with interaction (pre-post $\mathrm{x}$ group) were performed to assess the effects of acupuncture on elbow extension ROM at the onset of symptoms and 

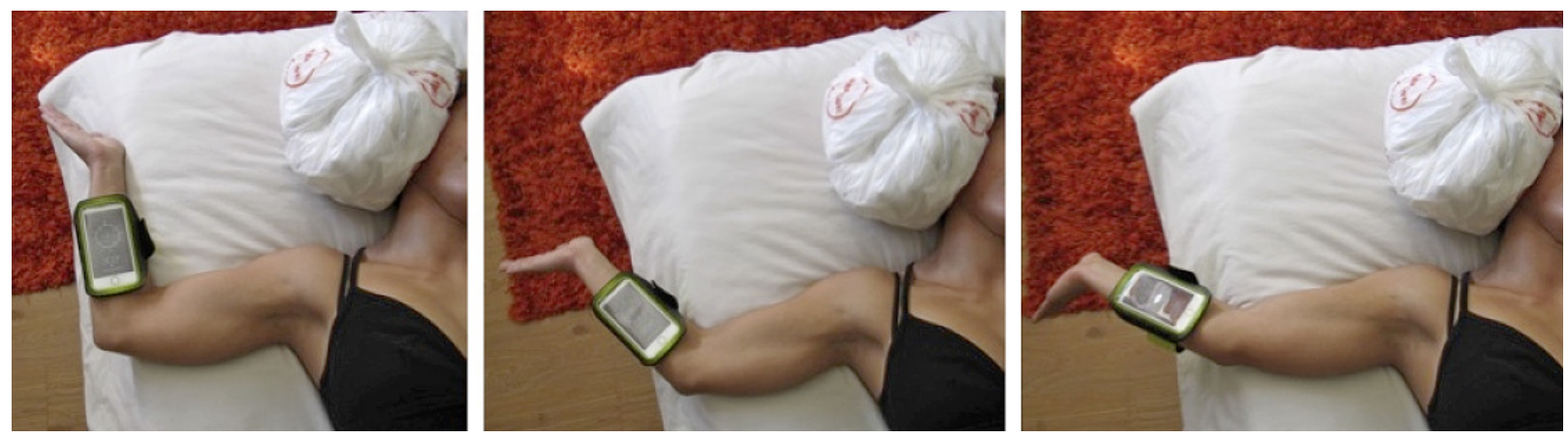

Fig. 1. Example of the MNT1 with measurement of elbow extension range of motion by means of a smartphone. Initial position (left frame), onset of symptoms (centre), and maximum tolerated elbow extension range of motion (right frame).

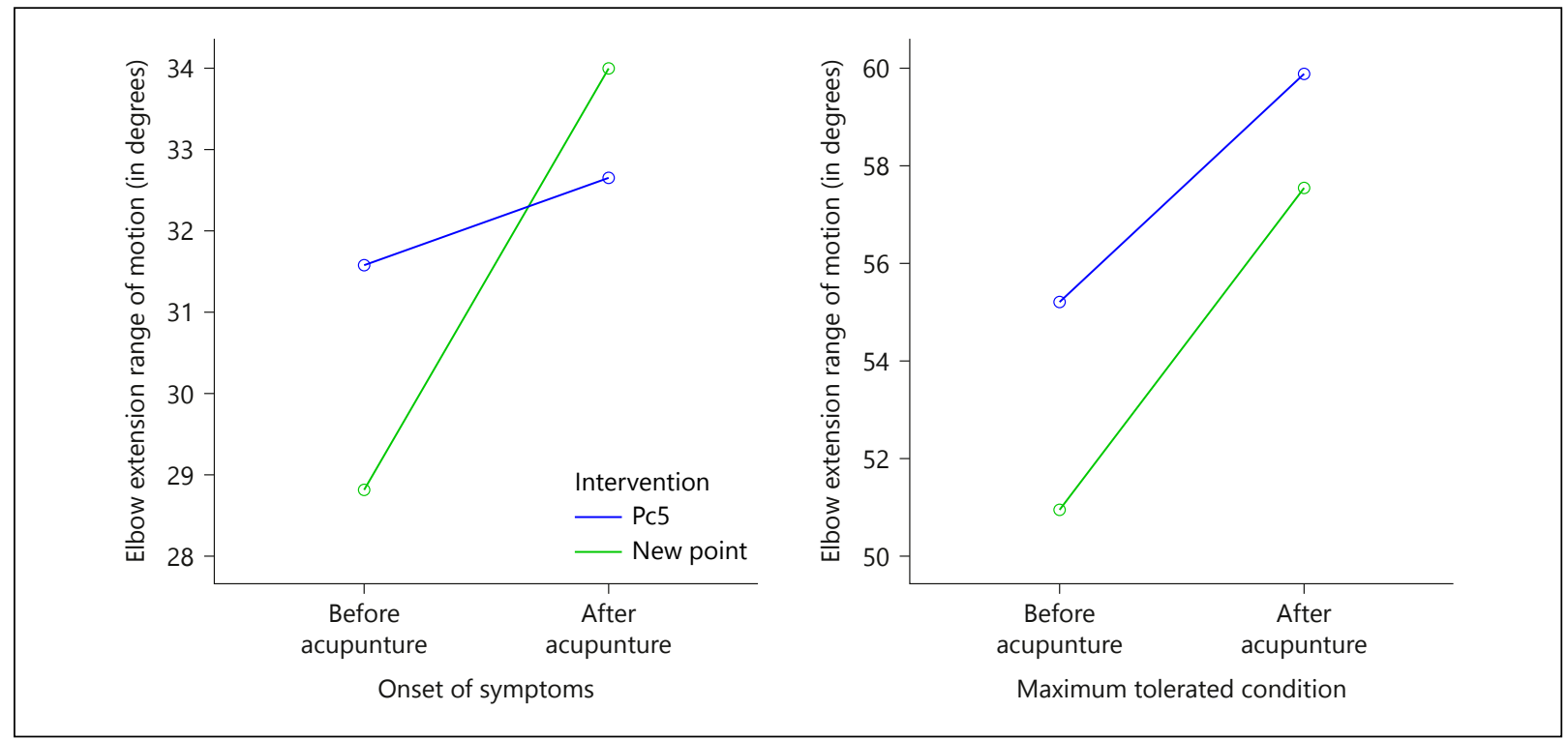

Fig. 2. Effects of acupuncture on elbow extension range of motion during the MNT1. Left frame, onset of symptoms, and right frame, maximum tolerated range of motion.

maximum tolerated point. Analyses were conducted using SPSS v22.0 (IBM Corp., Armonk, NY, USA) and significance level was set at $p<0.05$. Effect size $\left(\eta^{2}{ }_{p}\right)$ was calculated to complement inferential statistics.

Results: Acupuncture had a small (up to $6^{\circ}$ ) but significant effect on elbow extension ROM at both the onset of the symptoms $\left(p=0.029, \eta^{2}=0.154\right)$ and maximum tolerated point ( $p=0.002, \eta^{2}{ }_{p}=0.277$ ). No significant differences were found between groups at either the onset of the symptoms $\left(p=0.901, \eta^{2}=0.001\right)$ or the maximum tolerated point $(p=$ $0.585, \eta^{2}{ }_{p}=0.010$ ) (fig. 2).

Conclusion: Acupuncture increased the elbow extension ROM during the MNT1 in asymptomatic subjects, possibly due to reduced mechanossensitivity of the median nerve or altered pain perception. No acupoint proved to be better at improving the onset of symptoms and the maximum tolerated elbow extension ROM. Further studies are necessary to understand the clinical significance of these findings. 


\section{Acupuncture Treatment May Induce Immunoenhancing Effects by Increasing NK Cells Number in Patients with Colo-Rectal Cancer: A Randomized Pilot Study}

Irene Pais' ${ }^{1}$, Nuno Correia, ${ }^{7,11}$, Isabel Pimentel2, Maria José Teles ${ }^{3,8}$, Esmeralda Neves ${ }^{4}$, Júlia Vasconcelos ${ }^{4}$, Judite Guimarães ${ }^{4}$, Nancy Azevedo ${ }^{4}$, A. Moreira Pinto ${ }^{5}$, Jorge Machado ${ }^{1,9}$, Thomas Efferth ${ }^{6}$, Henry J. Greten, 1,10

${ }^{1}$ Abel Salazar Institute for Biomedical Sciences, University of Oporto, Porto, Portugal; ${ }^{2}$ Service of Oncology, Hospital Center of S. João, Oporto, Portugal; ${ }^{3}$ Service of Clinical Pathology, Hospital Center of S. João, Oporto, Portugal; ${ }^{4}$ Service of Clinical Pathology, Porto Hospital Center, Oporto, Portugal; ${ }^{5}$ Department of Oncology, Hospital Center of Vila Nova de Gaia/Espinho, Vila Nova de Gaia, Portugal; ${ }^{6}$ Department of Pharmaceutical Biology, Institute of Pharmacy and Biochemistry, Johannes Gutenberg University, Mainz, Germany; ${ }^{7}$ Emergency Department, Internal Medicine, Hospital Center of S. João, Oporto, Portugal; 8 ISPUP-EPI Unit, Institute of Public Health, University of Oporto, Portugal; ${ }^{9}$ Oporto Biomechanics Laboratory, Oporto, Portugal; ${ }^{10}$ Heidelberg School of Chinese Medicine, Heidelberg, Germany; ${ }^{11}$ Acupuncture Clinic, Alfena Private Hospital, Valongo, Portugal

Introduction: Colorectal cancer (CRC) is a major cause of death due to cancer worldwide. The functional state of the immune system has a major prognostic and predictive impact on the outcome of cancer patients. Natural killer cells (NKC) are a first line of defence against the metastatic spread of tumour cells and low NKC activity have been associated with higher stress levels. Acupuncture appears to modulate immunosuppressed or immunoactivated conditions through different mechanisms, including NKC activity, immunoglobulin production and activation of the complement system.

Objectives: To assess the effect of acupuncture and moxibustion (AcuMoxa) on the immune function of CRC patients, namely on NKC.

Methods: CRC patients recruited from Oncology Departments of two major hospital centers were randomized in two groups, experimental $(\mathrm{n}=9)$ and control (no intervention, $\mathrm{n}=$ 9) (fig. 1). In the experimental group, patients were submitted to AcuMoxa protocol following the Heidelberg Model of TCM (6 sessions/month). Blood samples were collected at baseline (T0) prior to chemotherapy and every once a week until next chemotherapy (CT) regimen (fig. 2). Questionnaires for assessment of quality of life (QoL CR-30, CR29) and anxiety and depression (HADS) were administered at the beginning (T0) and at the end of the study (T3).

Results: Differences among groups were statistically significant for WBC, ANC (fig. 3) and B cells (fig. 4) after 6 AcuMoxa treatments and for NKC after 2 AcuMoxa treatments (fig. 5). Patients' psychological status improved after acupuncture (fig. 6), along with improved QoL as well as a reduction of CT side effects.

Conclusion: AcuMoxa treatment showed immunoenhancing effects by increasing NKC number in patients with
Fig. 1. CONSORT flow of participants through the study.

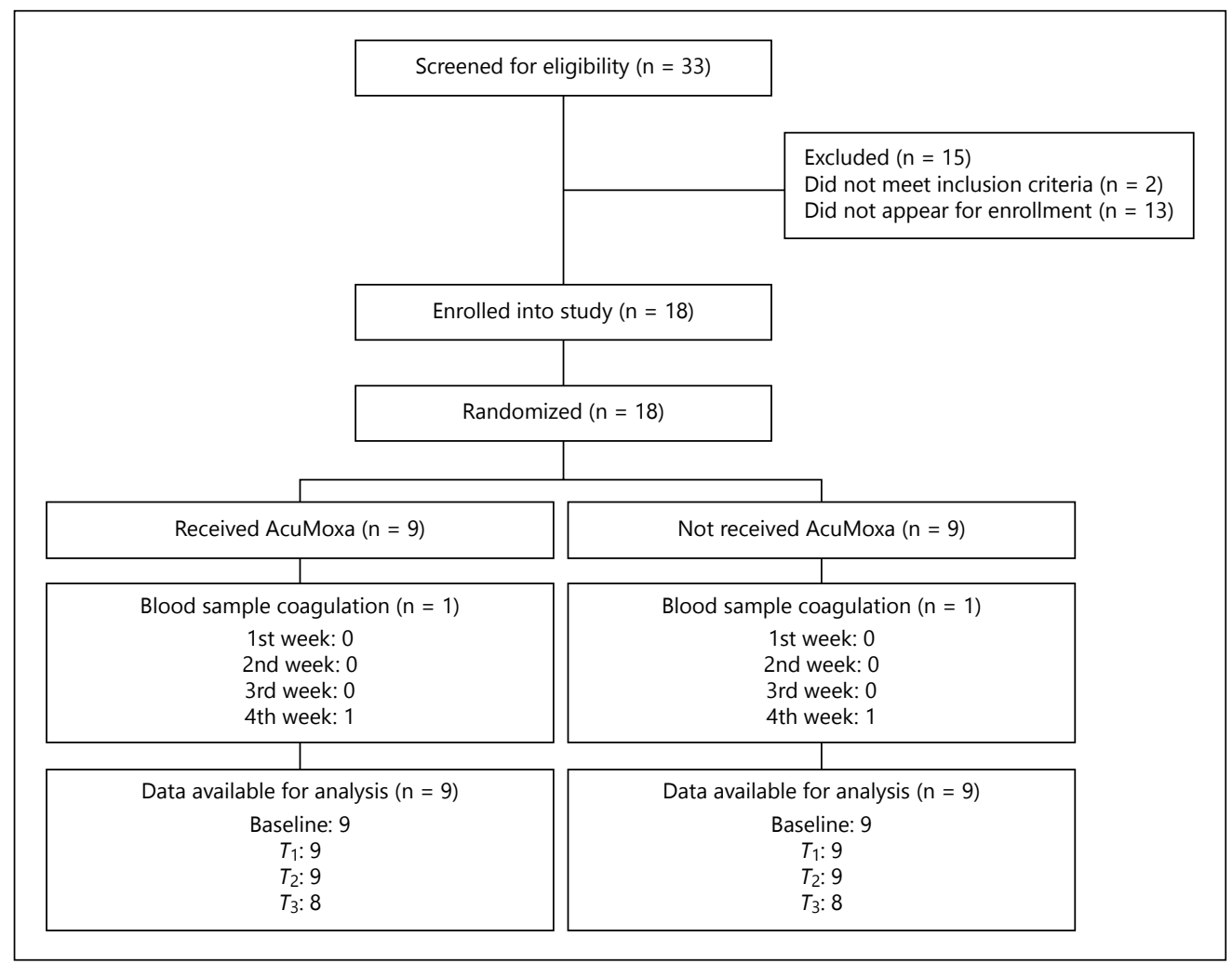


Fig. 2. Study flow chart. Black solid dots: time points of outcome measurements. Open circle: first chemotherapy day. Black diamonds: the primary endpoints of the study. Dashed lines: the expected changes, during chemotherapy, of white blood cells (WBC) and absolute neutrophil counts (ANC). Short, blue down arrows: acupuncture treatments. CBC, complete blood counts; NK, NK cells and subsets.

Fig. 3. WBC and ANC. Comparison between groups.
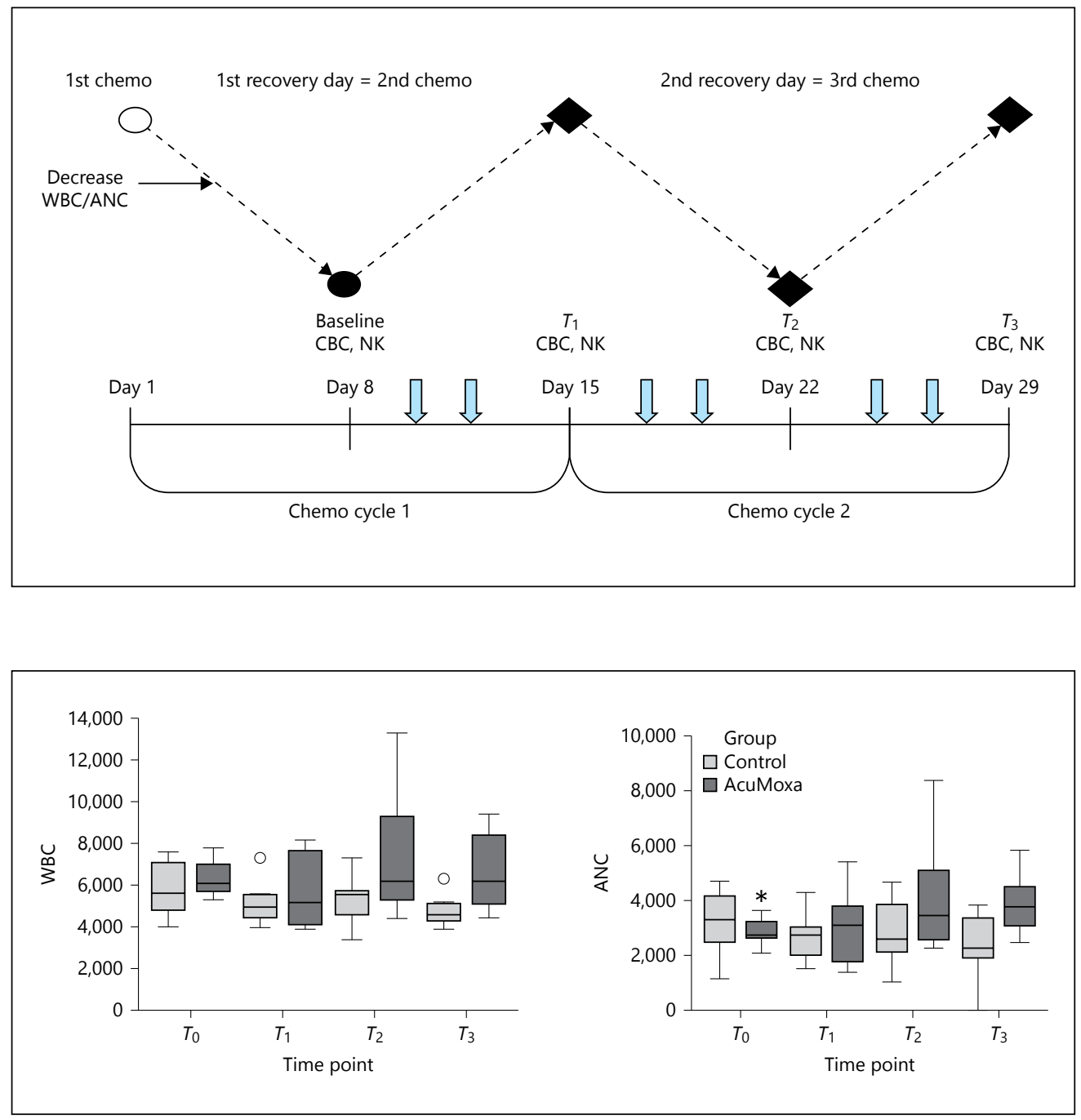

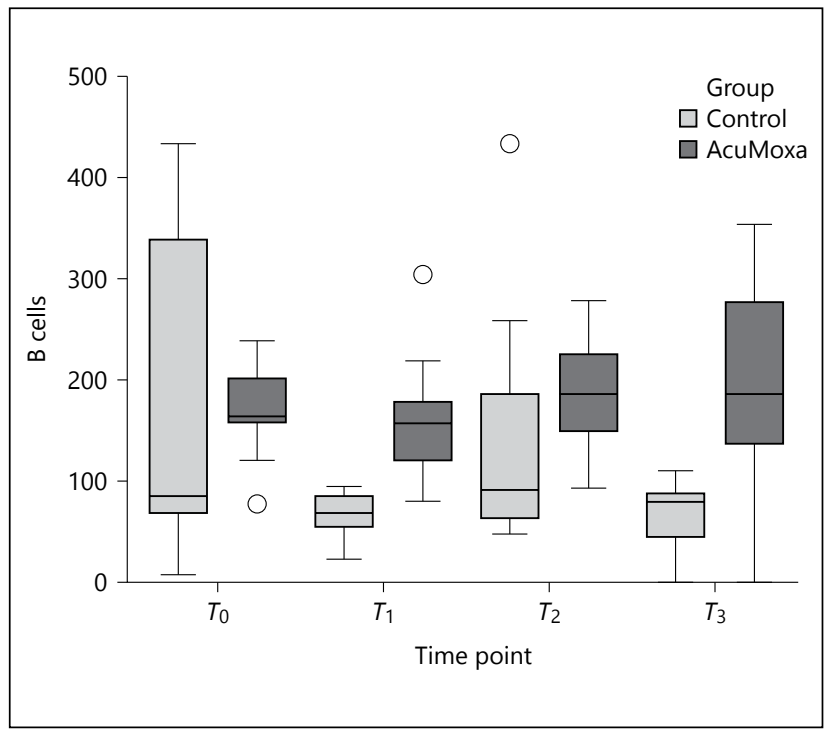

Fig. 4. B cells levels.

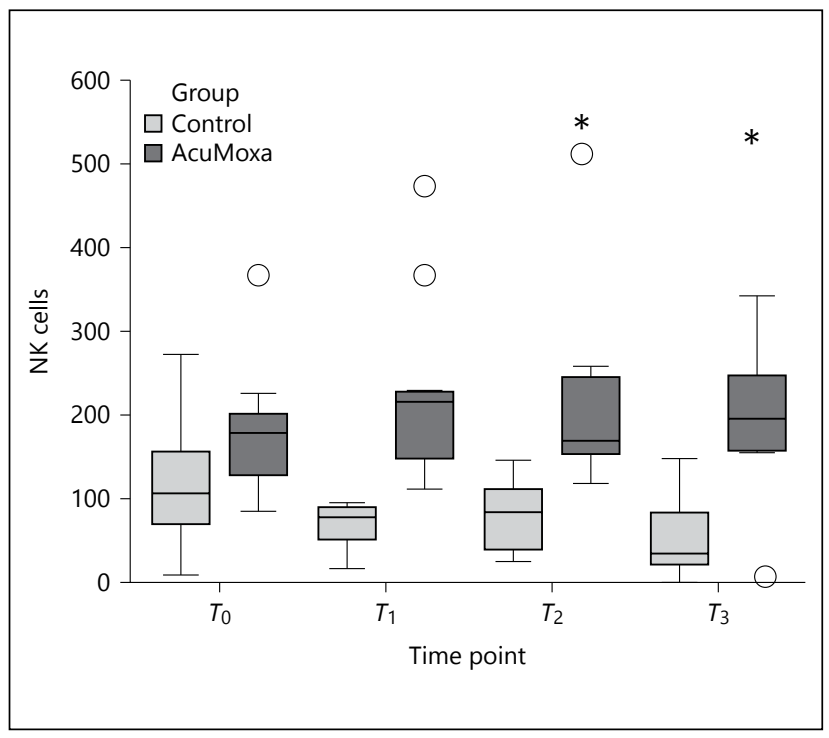

Fig. 5. Total NK cells counts. Comparison between groups. 
Fig. 6. Effect of AcuMoxo on the psychological status.

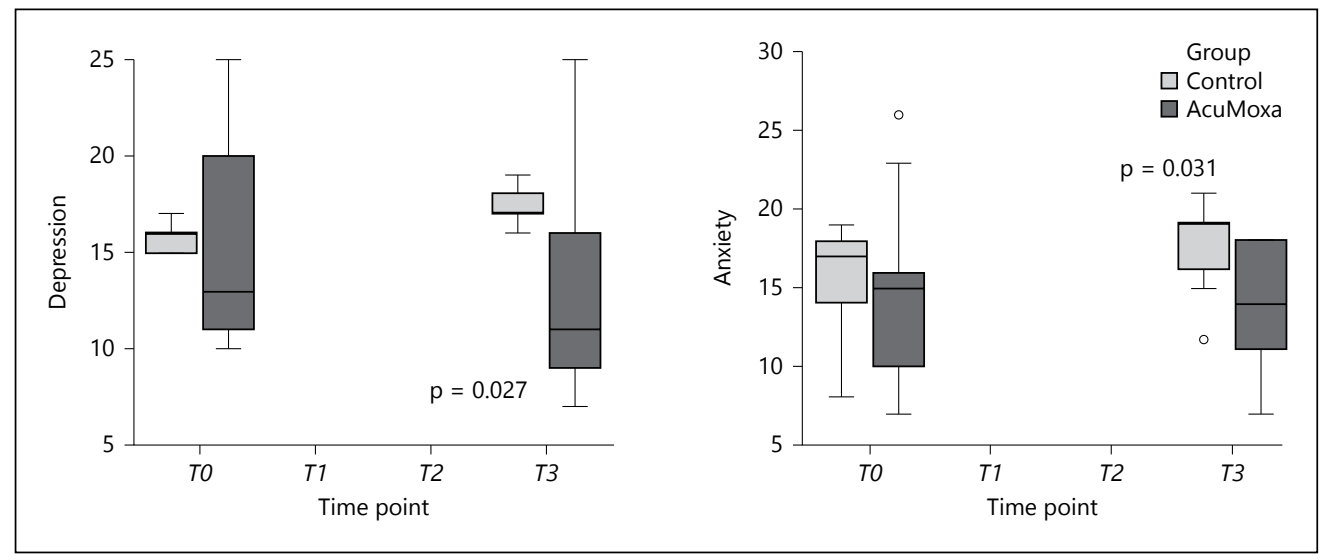

CRC, correlating with a better psychological status and a reduction of CT side effects. This study protocol proved to be feasible and safe, thus supporting a further larger and long term trial.

\section{Qigong Improves the Balance of Elderly People as Measured by Static and Dynamic Balance - Results from a Clinical Pre-Study} Liliana Sousa ${ }^{1}$, Mario Gonçalves ${ }^{1,2}$, Henry Greten ${ }^{1,2}$

${ }^{1}$ TCM Master Programme, Abel Salazar Institute for Biomedical Sciences, University of Porto, Porto, Portugal; ${ }^{2}$ Heidelberg School of Chinese Medicine, Heidelberg, Germany

Background: Falls are associated with serious morbidity among elderly and are one of the most leading causes of death among this population. The ability to maintain postural stability is the most relevant factor for fall prediction, both in community-dwelling as in institutionalised elderly populations. Qigong exercises have been reported in some studies to increase balance parameters and enhance the ability to tolerate unsteadiness.

Objective: The main objective with this study is to assess if Qigong exercises could improve balance parameters in the elderly. Further objectives are to evaluate parameters related to the loss of postural stability and the occurrence of falls, such as physical activity and fear of falling.

Methods: A randomized non-blinded controlled clinical trial was conducted. Institutionalized subjects, aged 60 yearsold or more, with history of falls were recruited from a nursing home and allocated into 2 groups: (a) Qigong group ( $\mathrm{n}=13$ ) and (b) control group (wait-list group $=8$ ). Qigong selftraining exercises were performed during 10 days. The main parameters measured were: (1) static balance by a balance platform; (2) the static balance by single leg stance and (3) the dynamic balance by the up and go test. Measurements were applied before and after the Qigong program to evaluate a possible improvement in balance. Physical activity and fear of falling were measured at baseline.

Results:

1. Balancecheck platforms:

a. There was a statistically improvement of $19.78 \%$ in the test on the foam surface with eyes closed in the Qigong group ( $\mathrm{p}$-value $=0.003$ ).

b. There were also improvements of $0.61 \%$ in the tests on normal surface with eyes closed and $1.78 \%$ on the foam surface with eyes open in the Qigong group although not statistically significant.

2. Single leg stance score test:

a. The score tend to improve in the Qigong group (though not statistically significant).

b. In control group this test score was worsened, indeed it was founded a statistically aggravation (p-value $=0.036)$.

c. Comparing Qigong group with the control group there was a statistically significant differences (p-value of 0.043 ) in the medians between the groups.

3. Up and go test score tend to improve in Qigong group after the intervention and worsen in the controls (not statistically significant).

Conclusion: Based on a sample of $13 / 8$, positive improvements were observed for static and dynamic balance parameters by the so-called White-ball exercises. These results are clinically significant since the elders were naïve to the intervention and they performed only a short-term course of Qigong. Assessment of the impact of a Qigong long-term programme on the risk of fall is needed. 


\section{Improving Neck Pain by Korean Hand Acupuncture in Neck Pain: Results from a Clinical Pre-Study}

Eva Teixeira ${ }^{1}$, Nuno Correia ${ }^{2,3,4}$, Maria João Santos ${ }^{1}$, Jorge Machado ${ }^{1}$, Henry J. Greten ${ }^{1,5}$

${ }^{1}$ TCM Master Programme, Abel Salazar Institute for Biomedical Sciences, University of Porto, Porto, Portugal; ${ }^{2}$ Internal Medicine, Emergency Department, University Hospital of São João, Porto, Portugal;

${ }^{3}$ Nursing School of Oporto, University of Porto; Porto, Portugal; ${ }^{4}$ Acupuncture Clinic, Alfena Private Hospital, Porto, Portugal; ${ }^{5}$ Heidelberg School of Chinese

Medicine, Heidelberg, Germany

Background: Neck pain is a very common musculoskeletal complaint among adults, affecting $50 \%$ of the population in some moment of their life-time. Its impact on quality of life is high and the efficacy of conventional treatments is limited. Korean hand acupuncture (KHA) is a new method of acupuncture largely used in Korea but less scientifically studied in the West. Lack of good quality randomized controlled trials has limited the evidence of the KHA treatments.

Objective: To design a randomized controlled clinical study which allows to scientifically study the acute effect of KHA for neck pain.

Methods: A clinical, experimental, randomized, controlled, single-blinded pre-study was developed. After approval from the Ethics Committee of the Abel Salazar Institute for Biomedical Sciences of the University of Porto, subjects with chronic mechanical neck pain and no previous contact with acupuncture were included. The experimental group (EG) was subject to KHA in hypersensitive points corresponding to neck area ('true' KHA). Hypersensitive points corresponding to the anterior surface of the thigh ('placebo' KHA) were needled in the control group (CG). Before (T0) and immediately after (T1) one 20 min session of KHA, pain (Visual Analogue Scale, VAS) and neck mobility (goniometry, GM) was measured in relation to the most painful neck movement.

Results: In the initial assessment of pain it wasn't observed statistical differences between the groups, experimental and control (VAS $p=0.682$ and goniometry $p=0.654$ ).

When comparing the differential percentile (T0-T1) of VAS and goniometry between both groups we can establish that:

1) Pain relieved 3 times more in the experimental group when compared with the control (median -3 vs. $-1.0 \mathrm{p}=$ 0.001).

2) The mobility improved 5 times more in the experimental group, with a significant difference between groups (median 11.95 vs. $2.5 \mathrm{p}<0.001$ ).

3) A significant correlation between pain and mobility improvement on the experimental group ( $\mathrm{p}<0.001)$.

Conclusion: This pilot project allowed the assessment of the feasibility of this study design. The data obtained subjects that KHA has a specific effect and can objectively improve neck pain.

In last analysis these results contribute for the scientific validation, underlying the theory of the hypersensitive points of KHA chosen. A long term study in KHA, with an adequate sample dimension and with an adequate measuring tool, could help to clarify the effect of KHA in this disease. 


\section{Author Index}

\section{Numbers refer to page numbers}

Amorim, P. 115

Anjos, A. 105, 108, 118

Azevedo, N. 124

Barros, F. 118

Barros, V. 102, 121

Barros, V.A. 110

Borges, G. 106

Brandão, L. 119

Brazkiewicz, F. 114

Cardoso, R. 111

Carvalho, L. 105

Chen, B. 99

Cho, T.H. 98

Cho, Z.-H. 91

Coelho, R. 115

Coimbra, D. 116

Correia, M. 102

Correia, N. 101, 102, 103, 106, 107,

$110,111,113,114,115,117,118$, $119,120,121,124,127$

Costa, A.R. 120

Costa, M. 106

Criado, M.B. 105

de Matos, L. 119

Doenitz, C. 114

Donitz, C.A. 118

Duarte, A. 111

Duarte, L. 112

Duarte, S. 101

Efferth, T. 114, 116, 118, 124

Eungpinichpong, W. 100

Fernandes, T. 105

Ferreira, C.A. 104

Ferreira, E. 101

Froeschen, P. 112, 116

Gao, X.-Y. 95

Goncalves, M. 116

Gonçalves, M. 109, 112, 126

Greten, H. 111, 113, 119, 121, 122,126
Greten, H.J. 92, 101, 102, 103, 104, $105,106,107,108,109,110,111$, $112,113,114,115,116,117,118$, $120,122,124,127$

Greten, T. 116, 118

Guimarães, J. 124

Hamza, F. 108

Hinzmann, M. 117

Ibar, K. 120

Jacinto, R. 121

Kreuzig, W. 92

Laranjeira, E. 111

Laranjeira, M. 108, 111, 117

Liça, N. 115

Litscher, D. 96

Litscher, G. 91

Liu, Z. 99

Lopes-Lima, M. 116

Loureiro, R. 107

Loureiro, R.P. 107, 108

Machado, J. 101, 102, 103, 104, 105, $106,107,108,109,110,111,112$, $113,114,115,116,117,120,124$, 127

Martins, S. 106

Matos, D. 110

Medeiros, A. 117

Mendes, P. 112

Morais, N. 122

Moreira, A. 122

Moreira, S. 104

Moreira Pinto, A. 124

Neves, E. 124

Ntritsou, V. 98

Oliveira, R. 109

Pais, I. 124

Paiva, C. 103
Paiva, C.A. 113

Pan, W. 97

Park, K.H. 94

Park, K.M. 98

Pimentel, I. 124

Pinho, P. 109

Pinto, K. 113

Quah-Smith, I. 96, 97

Ramos, B. 106

Rocha da Silva, M.H. 103

Sá, M. 111, 114

Santos, M.J. 101, 102, 103, 104, 105, $106,107,108,109,110,111,113$ $114,117,120,122,127$

Seca, S. 116

Seiça, A. 115

Silva, A.M. 102

Silva, K. 117

Silva, N. 115

Sousa, C. 103

Sousa, C.M. 116

Sousa, L. 126

Sousa, N. 117

Sousa, S. 103

Stürmer, J. 110, 117

Teixeira, E. 127

Teles, M.J. 124

Theodoratou, K. 93

Vasconcelos, J. 124

Vent, J. 114

Vieira, A. 110, 117

Wang, G.-J. 95

Wang, L.-P. 94

Wang, X. 100

Winck, J.C. 114

Yang, J. 100

Yoo, T.W. 94

Zhang, Z.-J. 93 